\title{
Derivative Couplings between Time-Dependent Density Functional Theory Excited States in the Random-Phase Approximation Based on Pseudo-Wavefunctions: Behavior around Conical Intersections
}

\author{
Qi Ou, Ethan C. Alguire, and Joseph E. Subotnik* \\ Department of Chemistry, University of Pennsylvania, Philadelphia, Pennsylvania 19104, United States
}

\begin{abstract}
In this paper, we present a formalism for derivative couplings between time-dependent density functional theory (TD-DFT) excited states within the randomphase approximation (RPA) using analytic gradient theory. Our formalism is based on a pseudo-wavefunction approach in a companion paper (DOI 10.1021/jp505767b), and can be checked against finite-difference overlaps. Our approach recovers the correct properties of derivative couplings around a conical intersection $(\mathrm{CI})$, which is a crucial prerequisite for any derivative coupling expression. As an example, we study the test case of protonated formaldimine $\left(\mathrm{CH}_{2} \mathrm{NH}_{2}^{+}\right)$.
\end{abstract}

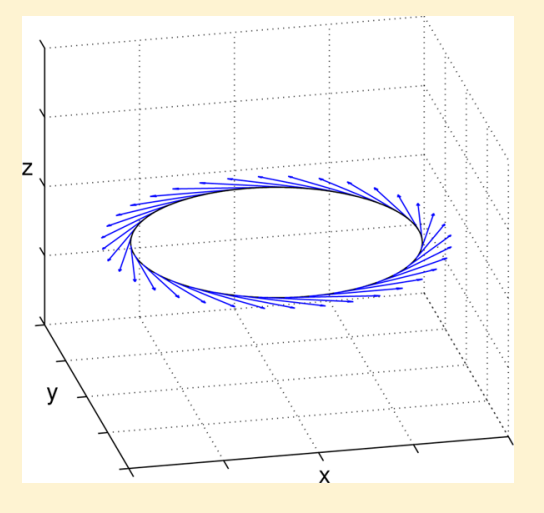

\section{INTRODUCTION}

The random-phase approximation (RPA) has been an extremely useful tool for calculating excited state properties and the oscillator strength of atoms and molecules since the $1960 \mathrm{~s}^{1-5}$ More recently, the application of RPA in timedependent density functional theory (TD-DFT/RPA) has played an increasingly important role in the field of theoretical chemistry for several reasons: (i) As a single-reference $a b$ initio method, TD-DFT/RPA is computationally affordable and sometimes retains relatively high accuracy. ${ }^{6,7}$ (ii) TD-DFT/ RPA is a size-consistent method which is able to give pure singlet and triplet states for closed-shell molecules. (iii) In contrast to TD-DFT within the Tamm-Dancoff approximation (TDA), TD-DFT/RPA maintains the Thomas-Reiche-Kuhn sum rule of the oscillator strengths by taking into account the $\mathrm{B}$ matrix in the TD-DFT working equation; ${ }^{9}$ as such, TD-DFT/ RPA gives improved results for transition moment calculations. ${ }^{8,10}$ For these reasons, despite its well-known triplet instability, ${ }^{8,11}$ TD-DFT/RPA is one of the most widely used approaches for modeling excited-state electronic structure. ${ }^{12-18}$

Recently, there has been a renewed interest in nonadiabatic dynamical transitions, as molecular photochemistry has gained renewed attention in the search for efficient solar energy capture. For these applications, the derivative coupling between excited states as well as the derivative coupling between the ground state and an excited state are necessary matrix elements for most theoretical approaches. To date, several researchers have investigated derivative couplings within TD-DFT and most of them have focused on the ground-excited state derivative couplings. Historically, the derivative coupling matrix elements between TD-DFT ground and excited states were first constructed by Chernyak and Mukamel. ${ }^{19}$ A similar method for real-time TD-DFT was developed by Baer and was applied to a molecular system $\left(\mathrm{H}+\mathrm{H}_{2}\right){ }^{20}$ Extensive calculations of TDDFT ground-excited state derivative couplings were then presented in both the plane-wave pseudopotential framework ${ }^{21-23}$ and the atomic orbital (AO) basis. ${ }^{24,25}$

The evaluation of TD-DFT derivative couplings is necessarily complicated by the fact that there are no rigorous static TD-DFT wavefunctions-TD-DFT is based on time-dependent response theories as opposed to a quantum chemistry theory based on a wavefunction ansatz. Despite this obstacle, however, TD-DFT derivative couplings should still meet certain benchmarks around a conical intersection $(\mathrm{CI})$ point: $(\mathrm{i})$ the magnitude of the derivative couplings should become enormous; (ii) the derivative coupling vectors should lie in the branching plane; (iii) the path integral of the derivative coupling should give Berry's phase. ${ }^{26-28} \mathrm{Hu}$ et al. have studied the behavior of the ground-excited state derivative coupling around various intersections, ${ }^{22,29-31}$ and they have found that the derivative coupling (as given by their modified linear response scheme within the TDA) recovers the correct Berry phase behavior. ${ }^{30,31}$

Looking forward, we are interested in the TD-DFT derivative couplings between excited states. Unlike the case of groundexcited state crossings, TD-DFT recovers the correct dimensionality of a CI branching plane for the case of excited-excited state crossings. ${ }^{32,33}$ A method for calculating the derivative coupling between TD-DFT excited states was proposed a few years ago by Tavernelli et al. ${ }^{34}$ based on Casida's ansatz, ${ }^{9}$ but the behavior around CIs was not fully investigated. To our

Special Issue: John R. Miller and Marshall D. Newton Festschrift

Received: June 10, 2014

Revised: July 30, 2014 
knowledge, in practice, Tavernelli et al. have focused on the TDA exclusively, rather than full TD-DFT/RPA. ${ }^{34-37}$ From the derivations that follow in section II, one can show that the Tavernelli formalism does not agree with our result, nor does the Tavernelli formalism satisfy the Chernyak-Mukamel equality. (More recently, while our article was under review, $\mathrm{Li}$ and Liu presented two abstract approaches for computing excited state derivative couplings which are applicable to TDDFT; the first is from a time-independent equation of motion (EOM) formalism, and the second is from time-dependent response theory. ${ }^{38}$ It can be shown that the final expression obtained in this work (eq 50) can be derived from $\mathrm{Li}$ and $\mathrm{Liu}$ using their time-independent EOM formalism to calculate particle-hole RPA derivate couplings.)

In the following sections, our goal is to derive explicit expressions for analytical derivative couplings between TD-DFT/RPA excited states and analyze the behavior of the TD-DFT/RPA derivative couplings around a CI. Analogous to the configuration interaction singles (CIS) and TD-DFT/TDA derivative couplings in our previous work, ${ }^{39,40}$ our theory for TD-DFT/ RPA derivative couplings is based upon direct differentiation. For full response TD-DFT/RPA (rather than TD-DFT/TDA), we use the pseudo-wavefunctions proposed and evaluated for the time-dependent Hartree-Fock (TD-HF) in ref 41. To justify our approach, first we will show in section $\mathrm{V}$ that our derivative couplings behave correctly around a CI. To our knowledge, the derivative couplings between TD-DFT/RPA excited states around a CI have not yet been investigated (nor has the corresponding Berry phase). Second, we will show in section VI that our final answer satisfies the ChernyakMukamel equality near a crossing (again drawing on the results of ref 41); thus, our results are mostly consistent with response theory.

An outline of this paper is as follows. In section II, our analytic gradient formalism for TD-DFT/RPA derivative couplings will be presented. In section III, we compare our results versus the finite-difference data. In section IV, using a simple model of four diabatic states, we analyze for TD-DFT/RPA (or TD-HF) derivative coupling vectors around a CI. In section V, we consider the specific case of protonated formaldimine and we examine the CI between the first and second excited singlet states; we show that our derivative couplings lie in the correct branching plane and recover the Berry phase exactly. In section VI, we compare our derivative coupling expression with the Chernyak-Mukamel equality and transition densities from time-dependent response theory.

Unless otherwise specified, we use lowercase latin letters to denote spin molecular orbitals (MO) $(a, b, c, d$ for virtual orbitals, $i, j, k, l, m$ for occupied orbitals, and $p, q, r, s, w$ for arbitrary orbitals) and Greek letters $(\alpha, \beta, \gamma, \delta, \lambda, \sigma, \mu, \nu)$ to denote AOs. The RPA excited states are denoted by $\Psi$ (with uppercase latin indices $I, J$ ).

\section{ANALYTIC DERIVATION FOR TD-DFT/RPA DERIVATIVE COUPLINGS}

A. TD-DFT/RPA Eigenvalue Equation. Before addressing derivative couplings between excited states, we first review the TD-DFT/RPA eigenvalue equations and establish the necessary notations. As discussed in ref 8 , the non-Hermitian eigenvalue TD-DFT/RPA equation can be written as

$$
\left(\begin{array}{cc}
\mathbf{A} & \mathbf{B} \\
-\mathbf{B} & -\mathbf{A}
\end{array}\right)\left(\begin{array}{l}
\mathbf{X}^{I} \\
\mathbf{Y}^{I}
\end{array}\right)=E_{I}\left(\begin{array}{c}
\mathbf{X}^{I} \\
\mathbf{Y}^{I}
\end{array}\right)
$$

for excitation amplitudes $\mathbf{X}^{I}$ and $\mathbf{Y}^{I}$, and excitation energy $E_{I}$ of TD-DFT/RPA excited state $I$. The orthogonality condition of TD-DFT/RPA excitation amplitudes for any two excited states $I$ and $J$ is

$$
\left(\begin{array}{ll}
\mathbf{X}^{I^{\dagger}} & -\mathbf{Y}^{I \dagger}
\end{array}\right)\left(\begin{array}{l}
\mathbf{X}^{J} \\
\mathbf{Y}^{J}
\end{array}\right)=\mathbf{X}^{I \dagger} \mathbf{X}^{J}-\mathbf{Y}^{I \dagger} \mathbf{Y}^{J}=\delta_{I J}
$$

Multiplying $\left(\mathrm{X}^{\dagger \dagger}-\mathrm{Y}^{\dagger \dagger}\right)$ on both sides of eq 1 gives

$$
\begin{aligned}
\left(\begin{array}{ll}
\mathbf{X}^{{ }^{\dagger}}-\mathbf{Y}^{J \dagger}
\end{array}\right)\left(\begin{array}{cc}
\mathbf{A} & \mathbf{B} \\
-\mathbf{B} & -\mathbf{A}
\end{array}\right)\left(\begin{array}{l}
\mathbf{X}^{I} \\
\mathbf{Y}^{I}
\end{array}\right) & =\mathbf{X}^{J^{\dagger}} \mathbf{A} \mathbf{X}^{I}+\mathbf{Y}^{J \dagger} \mathbf{A} \mathbf{Y}^{I} \\
+ & \mathbf{X}^{\dagger \dagger} \mathbf{B} \mathbf{Y}^{I}+\mathbf{Y}^{J \dagger} \mathbf{B} \mathbf{X}^{I} \\
& =\delta_{I J} E_{I}
\end{aligned}
$$

The matrix elements of $\mathbf{A}$ and $\mathbf{B}$ are given by

$$
\begin{aligned}
A_{i a j b} & =\left\langle\Phi_{i}^{a}\left|O_{\mathrm{KS}}\right| \Phi_{j}^{b}\right\rangle \\
& =\delta_{i j} F_{a b}-\delta_{a b} F_{i j}+\delta_{i j} \delta_{a b} E_{\mathrm{DFT}}+\Omega_{a j i b} \\
B_{i a j b} & =\left\langle\Phi_{i j}^{a b}\left|O_{\mathrm{KS}}\right| \Phi_{\mathrm{DFT}}\right\rangle \\
& =\Omega_{a b i j}
\end{aligned}
$$

where $O_{\mathrm{KS}}$ is the Kohn-Sham response operator. In secondquantized and antisymmetrized form (with physicists' notation for the two-electron integrals ${ }^{42}$ ), $O_{\mathrm{KS}}$ can be represented as

$$
\begin{aligned}
O_{\mathrm{KS}}= & \sum_{p q} F_{p q} a_{p}^{\dagger} a_{q}+\sum_{c d k l}\left[\Omega_{c l k d} a_{c}^{\dagger} a_{k} a_{l}^{\dagger} a_{d}\right. \\
& \left.+\Omega_{c d k l}\left(a_{c}^{\dagger} a_{d}^{\dagger} a_{l} a_{k}+a_{l}^{\dagger} a_{k}^{\dagger} a_{c} a_{d}\right)\right]
\end{aligned}
$$

where $F_{p q}$ is the Fock matrix, and $\Omega_{p q s}$ is the two-electron effective operator in DFT:

$$
\begin{aligned}
& F_{p q}=h_{p q}+\sum_{m} \Pi_{p m q m} \\
& \Omega_{p q s r}=\Pi_{p q s r}+\omega_{p q s r}
\end{aligned}
$$

The diagonal entries of the Fock matrix $F_{p p} \equiv \varepsilon_{p}$ are the usual Kohn-Sham orbital energies, $h_{p q}^{0}$ is the matrix element of the kinetic energy plus the external potential (eq 12), and $g_{p q}$ is the first derivative of the $x c$ functional $f_{x c}$ (eq 13). ${ }^{43}$ The sum of $h_{p q}^{0}$ and $g_{p q}$ gives the one-electron effective operator $h_{p q}$ (eq 14).

$$
\begin{aligned}
& h_{p q}^{0} \equiv\left\langle p\left|h^{0}\right| q\right\rangle \\
& g_{p q} \equiv \sum_{p q} \int \mathrm{d} \mathbf{r} \phi_{p}(\mathbf{r}) \frac{\partial f_{x c}}{\partial \rho(\mathbf{r})} \phi_{q}(\mathbf{r}) \\
& h_{p q} \equiv h_{p q}^{0}+g_{p q}
\end{aligned}
$$

$\Pi_{p q s r}$ is the Coulomb term plus whatever fraction of HartreeFock exchange is included in the DFT functional $\left(c_{\mathrm{HF}}\right.$ in eq 15), and $\omega_{p q s r}$ is the second derivative of the $x c$ functional (eq 16). The sum of $\Pi_{p q s r}$ and $\omega_{p q s r}$ gives $\Omega_{p q s r}$ (eq 11).

$$
\Pi_{p q s r} \equiv\langle p q \mid s r\rangle-c_{\mathrm{HF}}\langle p q \mid r s\rangle
$$




$$
\begin{aligned}
\omega_{p q s r} & \equiv\left\langle p q\left|f_{x c}^{\prime \prime}\right| s r\right\rangle \\
& =\sum_{p q s r} \int \operatorname{dr} \phi_{p}(\mathbf{r}) \phi_{q}(\mathbf{r}) \frac{\partial^{2} f_{x c}}{\partial \rho(\mathbf{r})^{2}} \phi_{r}(\mathbf{r}) \phi_{s}(\mathbf{r})
\end{aligned}
$$

With the definitions above, $O_{\mathrm{KS}}$ can be rewritten as

$$
\begin{aligned}
O_{\mathrm{KS}}= & \sum_{p q}\left(h_{p q}^{0}+g_{p q}+\sum_{m} \Pi_{p m q m}\right) a_{p}^{\dagger} a_{q} \\
& +\sum_{c d k l}\left[\left(\Pi_{c l k d}+\omega_{c l k d}\right) a_{c}^{\dagger} a_{k} a_{l}^{\dagger} a_{d}\right. \\
& \left.+\left(\Pi_{c d k l}+\omega_{c d k l}\right)\left(a_{c}^{\dagger} a_{d}^{\dagger} a_{l} a_{k}+a_{l}^{\dagger} a_{k}^{\dagger} a_{c} a_{d}\right)\right]
\end{aligned}
$$

B. The "Brute Force" Expression for TD-DFT/RPA Derivative Couplings. In ref 41 , we defined TD-HF pseudowavefunctions and we showed that the derivative coupling between $\Psi_{I}$ and $\Psi_{J}$ obtained by direct differentiation can be written as

$$
\begin{aligned}
\left\langle\Psi_{I} \mid \Psi_{J}^{[Q]}\right\rangle= & \sum_{i j a b}\left(X_{i}^{I a} X_{j}^{J b}-Y_{i}^{I a} Y_{j}^{J b}\right)\left\langle\Phi_{i}^{a} \mid \Phi_{j}^{b[Q]}\right\rangle \\
& +\sum_{i a}\left(X_{i}^{I a} X_{i}^{J a[Q]}-Y_{i}^{I a} Y_{i}^{J a[Q]}\right)
\end{aligned}
$$

We now treat TD-DFT/RPA as a natural extension of TD-HF, and the goal of this paper is to evaluate eq 18 for TD-DFT/RPA derivative couplings. We begin with the second term in eq 18. The first step is to take the derivative on each side of eqs 3 and 4 (for $I \neq J$ )

$$
\begin{aligned}
0= & \sum_{i j a b}\left\{X_{i}^{I a} A_{i a j b}^{[Q]} X_{j}^{J b}+Y_{i}^{I a} A_{i a j b}^{[Q]} Y_{j}^{J b}+X_{i}^{I a} B_{i a j b}^{[Q]} Y_{j}^{J b}\right. \\
& +Y_{i}^{I a} B_{i a j b}^{[Q]} X_{j}^{J b}+X_{i}^{I a[Q]} A_{i a j b} X_{j}^{J b}+Y_{i}^{I a[Q]} A_{i a j j} Y_{j}^{J b} \\
& +X_{i}^{I a[Q]} B_{i a j b} Y_{j}^{J b}+Y_{i}^{I a[Q]} B_{i a j b} X_{j}^{J b}+X_{i}^{I a} A_{i a j b} X_{j}^{J b[Q]} \\
& \left.+Y_{i}^{I a} A_{i a j b} Y_{j}^{J b[Q]}+X_{i}^{I a} B_{i a j b} Y_{j}^{J b[Q]}+Y_{i}^{I a} B_{i a j b} X_{j}^{J b[Q]}\right\}
\end{aligned}
$$

From eq 1, one finds that

$$
\begin{aligned}
& \sum_{i j a b} A_{i a j b} X_{j}^{I b}+B_{i a j b} Y_{j}^{I b}=\sum_{i a} E_{I} X_{i}^{I a} \\
& \sum_{i j a b} B_{i a j b} X_{j}^{I b}+A_{i a j b} Y_{j}^{I b}=-\sum_{i a} E_{I} Y_{i}^{I a}
\end{aligned}
$$

Inserting eqs 20 and 21 into eq 19, one has

$$
\begin{aligned}
\sum_{i a} & \left(X_{i}^{I a} X_{i}^{J a[Q]}-Y_{i}^{I a} Y_{i}^{J a[Q]}\right) \\
= & \frac{1}{E_{J}-E_{I}} \sum_{i j a b}\left\{X_{i}^{I a} A_{i a j b}^{[Q]} X_{j}^{J b}+Y_{i}^{I a} A_{i a j b}^{[Q]} Y_{j}^{J b}+X_{i}^{I a} B_{i a j b}^{[Q]} Y_{j}^{J b}\right. \\
& \left.+Y_{i}^{I a} B_{i a j b}^{[Q]} X_{j}^{J b}\right\}
\end{aligned}
$$

Now, we replace the second term of eq 18 by the result of eq 22 and the derivative coupling expression becomes

$$
\begin{aligned}
\left\langle\Psi_{I} \mid \Psi_{J}^{[Q]}\right\rangle= & \sum_{i j a b}\left(X_{i}^{I a} X_{j}^{J b}-Y_{i}^{I a} Y_{j}^{J b}\right)\left\langle\Phi_{i}^{a} \mid \Phi_{j}^{b[Q]}\right\rangle \\
& +\frac{1}{E_{J}-E_{I}} \sum_{i j a b}\left\{X_{i}^{I a} A_{i a j b}^{[Q]} X_{j}^{J b}\right. \\
& +Y_{i}^{I a} A_{i a j b}^{[Q]} Y_{j}^{J b}+X_{i}^{I a} B_{i a j b}^{[Q]} Y_{j}^{J b} \\
& \left.+Y_{i}^{I a} B_{i a j b}^{[Q]} X_{j}^{J b}\right\} \\
= & \frac{1}{E_{J}-E_{I}} \sum_{i j a b}\left\{\left(X_{i}^{I a} X_{j}^{J b}+Y_{i}^{I a} Y_{j}^{J b}\right) A_{i a j b}^{[Q]}\right. \\
& \left.+\left(X_{i}^{I a} Y_{j}^{J b}+Y_{i}^{I a} X_{j}^{J b}\right) B_{i a j b}^{[Q]}\right\} \\
& -\sum_{i a b}\left(X_{i}^{I a} X_{i}^{J b}-Y_{i}^{I a} Y_{i}^{J b}\right) O_{b a}^{R[Q]} \\
& -\sum_{i j a}\left(X_{i}^{I a} X_{j}^{J a}-Y_{i}^{I a} Y_{j}^{J a}\right) O_{j i}^{R[Q]}
\end{aligned}
$$

where we define the "right" spin-orbital derivative overlap

$$
\begin{aligned}
O_{p q}^{R[Q]} & \equiv\left\langle p \mid q^{[Q]}\right\rangle \\
& =\left(\sum_{\mu} C_{\mu p}\langle\mu|\right)\left(\sum_{\nu}|\nu\rangle C_{\nu q}^{[Q]}+\sum_{\nu}\left|\nu^{[Q]}\right\rangle C_{\nu q}\right) \\
& =\sum_{\mu \nu} C_{\mu p} S_{\mu \nu} C_{\nu q}^{[Q]}+\sum_{\mu \nu} C_{\mu p} S_{\mu \nu}^{R[Q]} C_{\nu q}
\end{aligned}
$$

Here $C_{\mu p}$ denotes the MO coefficients; $S_{\mu \nu} \equiv\langle\mu \mid \nu\rangle$ is the atomic orbital overlap, and $S_{\mu \nu}^{R[Q]} \equiv\left\langle\mu \mid \nu^{[Q]}\right\rangle$ is the right derivative of the overlap. The detailed derivation of $O_{p q}^{R[Q]}$ can be found in ref 39 , which leads to

$$
\begin{aligned}
O_{p q}^{R[Q]} & =\sum_{\mu \nu} C_{\mu p}\left(S_{\mu \nu}^{R[Q]}-\frac{1}{2} S_{\mu \nu}^{[Q]}\right) C_{\nu q}-\Theta_{p q}^{[Q]} \\
& =\sum_{\mu \nu} C_{\mu p} S_{\mu \nu}^{A[Q]} C_{\nu q}-\Theta_{p q}^{[Q]}
\end{aligned}
$$

where $\Theta$ is the occupied-to-virtual rotation angle matrix and $\mathbf{S}^{A[Q]}$ is defined as $\mathbf{S}^{A[Q]} \equiv \mathbf{S}^{R[Q]}-{ }_{1}^{1}{ }_{2} \mathbf{S}^{[Q]}$.

The derivatives of $\mathbf{A}$ and $\mathbf{B}$ in eq 24 can be written as

$$
\begin{aligned}
& A_{i a j b}^{[Q]}=\Omega_{a j i b}^{[Q]}+\delta_{i j} F_{a b}^{[Q]}-\delta_{a b} F_{i j}^{[Q]}+\delta_{i j} \delta_{a b} E_{\mathrm{DFT}}^{[Q]} \\
& B_{i a j b}^{[Q]}=\Omega_{a b i j}^{[Q]}
\end{aligned}
$$

As we did in our recent paper on TD-DFT/TDA derivative couplings, ${ }^{40}$ we make the following definitions for various types of density matrices:

(1) The general density matrices:

$$
\begin{aligned}
& P_{\mu \nu}=\sum_{m} C_{\mu m} C_{\nu m} \\
& \tilde{P}_{\mu \nu}=\sum_{p} C_{\mu p} C_{\nu p}=P_{\mu \nu}+\sum_{a} C_{\mu a} C_{\nu a}
\end{aligned}
$$

Note that we may express the real-space density as $\rho(\mathbf{r})=$ $\sum_{\mu \nu} P_{\mu \nu} \phi_{\mu}(\mathbf{r}) \phi_{\nu}(\mathbf{r})$

(2) The RPA excitation-amplitude matrices:

$$
\begin{aligned}
& R_{\mu \nu}^{X I}=\sum_{i a} C_{\mu a} X_{i}^{I a} C_{\nu i} \\
& R_{\mu \nu}^{Y I}=\sum_{i a} C_{\mu a} Y_{i}^{I a} C_{\nu i}
\end{aligned}
$$


(3) The generalized difference-density matrix:

$$
\begin{aligned}
D_{\mu \nu}^{I J}= & \sum_{i a b} C_{\mu a}\left(X_{i}^{I a} X_{i}^{J b}+Y_{i}^{J a} Y_{i}^{I b}\right) C_{\nu b} \\
& -\sum_{i j a} C_{\mu i}\left(X_{i}^{J a} X_{j}^{I a}+Y_{i}^{I a} Y_{j}^{J a}\right) C_{\nu j}
\end{aligned}
$$

Using the expressions for $\mathbf{\Omega}^{[Q]}$ and $\mathbf{F}^{[Q]}$ in refs 39 and 40, and plugging those expressions into $A_{i a j b}^{[Q]}$ and $B_{i a j b}^{[Q]}$, we find this lengthy expression for the TD-DFT/RPA derivative coupling:

$$
\begin{aligned}
& \left\langle\Psi_{I} \mid \Psi_{J}^{[Q]}\right\rangle=\sum_{i j a b} \frac{\left(X_{i}^{I a} X_{j}^{J b}+Y_{i}^{I a} Y_{j}^{J b}\right)}{E_{J}-E_{I}}\left\{\sum_{\mu \nu \lambda \sigma} C_{\mu a} C_{\nu j} \Omega_{\mu \nu \lambda \sigma}^{[Q]} C_{\lambda i} C_{\sigma b}\right. \\
& -\frac{1}{2} \sum_{\alpha \beta w} C_{\alpha w} S_{\alpha \beta}^{[Q]}\left[C_{\beta a} \Omega_{w j i b}+C_{\beta j} \Omega_{a w i b}+C_{\beta i} \Omega_{a j w b}\right. \\
& \left.+C_{\beta b} \Omega_{a j i w}\right]+\sum_{k} \Omega_{k j i b} \Theta_{a k}^{[Q]}+\sum_{c} \Omega_{a c i b} \Theta_{j c}^{[Q]} \\
& \left.+\sum_{c} \Omega_{a j c b} \Theta_{i c}^{[Q]}+\sum_{k} \Omega_{a j i k} \Theta_{b k}^{[Q]}\right\} \\
& +\sum_{i a b} \frac{\left(X_{i}^{I a} X_{i}^{J b}+Y_{i}^{I a} Y_{i}^{J b}\right)}{E_{J}-E_{I}} \times\left\{\sum_{\mu \nu} C_{\mu a}{ }_{\mu \nu}^{[Q]} C_{\nu b}\right. \\
& +\sum_{\mu \nu \lambda \sigma m} C_{\mu a} C_{\nu m} \Pi_{\mu \nu \lambda \sigma}^{[Q]} C_{\lambda b} C_{\sigma m}-\frac{1}{2}\left(\varepsilon_{a} \sum_{\alpha \beta} C_{\alpha a} S_{\alpha \beta}^{[Q]} C_{\beta b}\right. \\
& \left.+\varepsilon_{b} \sum_{\alpha \beta} C_{\alpha b} S_{\alpha \beta}^{[Q]} C_{\beta a}\right)-\frac{1}{2} \sum_{\alpha \beta m w} C_{\alpha w} S_{\alpha \beta}^{[Q]} C_{\beta m} \\
& \left.\left(\Pi_{a w b m}+\Pi_{a m b w}\right)-\sum_{m c} \Theta_{c m}^{[Q]}\left(\Pi_{a c b m}+\Pi_{a m b c}\right)\right\} \\
& -\sum_{i j a} \frac{\left(X_{i}^{I a} X_{j}^{J a}+Y_{i}^{I a} Y_{j}^{J a}\right)}{E_{J}-E_{I}}\left\{\sum_{\mu \nu} C_{\mu i} h_{\mu \nu}^{[Q]} C_{\nu j}\right. \\
& +\sum_{\mu \nu \lambda \sigma m} C_{\mu i} C_{\nu m} \Pi_{\mu \nu \lambda \sigma}^{[Q]} C_{\lambda j} C_{\sigma m}-\frac{1}{2}\left(\varepsilon_{i} \sum_{\alpha \beta} C_{\alpha i} S_{\alpha \beta}^{[Q]} C_{\beta j}\right. \\
& \left.+\varepsilon_{j} \sum_{\alpha \beta} C_{\alpha j} S_{\alpha \beta}^{[Q]} C_{\beta i}\right)-\frac{1}{2} \sum_{\alpha \beta m w} C_{\alpha w} S_{\alpha \beta}^{[Q]} C_{\beta m}\left(\Pi_{i w j m}\right. \\
& \left.\left.+\Pi_{i m j w}\right)-\sum_{m c} \Theta_{c m}^{[Q]}\left(\Pi_{i c j m}+\Pi_{i m j c}\right)\right\} \\
& +\sum_{i j a b} \frac{\left(X_{i}^{I a} Y_{j}^{J b}+X_{i}^{I a} Y_{j}^{J b}\right)}{E_{J}-E_{I}}\left\{\sum_{\mu \nu \lambda \sigma} C_{\mu a} C_{\nu b} \Omega_{\mu \nu \lambda \sigma}^{[Q]} C_{\lambda i} C_{\sigma j}\right. \\
& -\frac{1}{2} \sum_{\alpha \beta w} C_{\alpha w} S_{\alpha \beta}^{[Q]}\left[C_{\beta a} \Omega_{w b i j}+C_{\beta b} \Omega_{a w i j}+C_{\beta i} \Omega_{a b w j}\right. \\
& \left.+C_{\beta j} \Omega_{a b i w}\right]+\sum_{k} \Omega_{k b i j} \Theta_{a k}^{[Q]}+\sum_{c} \Omega_{a b i c} \Theta_{j c}^{[Q]} \\
& \left.+\sum_{c} \Omega_{a b c j} \Theta_{i c}^{[Q]}+\sum_{k} \Omega_{a k i j} \Theta_{b k}^{[Q]}\right\} \\
& -\sum_{\mu \nu} S_{\mu \nu}^{A[Q]}\left[\sum_{i a b}\left(X_{i}^{I a} X_{i}^{J b}-Y_{i}^{I a} Y_{i}^{J b}\right) C_{\mu b} C_{\nu a}\right. \\
& \left.+\sum_{i j a}\left(X_{i}^{I a} X_{j}^{J a}-Y_{i}^{I a} Y_{j}^{J a}\right) C_{\mu j} C_{\nu i}\right]
\end{aligned}
$$

In eq 37 , the terms in the first three braces come from $\mathbf{A}^{[Q]}$, the last brace comes from $\mathbf{B}^{[Q]}$, and the final line is contributed by $\mathbf{O}^{R[Q]}$. Note that the orbital rotations within the occupied and virtual subspaces $\left(\Theta_{a b}\right.$ and $\left.\Theta_{i j}\right)$ disappear and are absent in eq 37 (just as for CIS and TD-DFT/TDA ${ }^{39,40}$ ). These terms vanish because TD-DFT is invariant to one's choice of occupied and virtual orbitals; as such, $\Theta_{a b}$ and $\Theta_{i j}$ cannot contribute to the final answer.

In order to get our final expression into the AO basis, a few more definitions are needed for the $x c$ functional derivatives; these definitions are identical with those for TD-DFT/TDA. First, the first derivative of the $x c$ functional $\mathbf{g}^{[Q]}$ can be decomposed in the $\mathrm{AO}$ representation as

$$
g_{\mu \nu}^{[Q]} \equiv \tilde{g}_{\mu \nu}^{[Q]}+g_{\mu \nu}^{Y[Q]}
$$

where

$$
\begin{aligned}
\tilde{g}_{\mu \nu}^{[Q]} \equiv & \int \mathrm{d} \mathbf{r} \phi_{\mu}(\mathbf{r}) \frac{\partial f_{x c}}{\partial \rho(\mathbf{r})} \phi_{\nu}(\mathbf{r})+\int \mathrm{d} \mathbf{r} \frac{\partial f_{x c}}{\partial \rho(\mathbf{r})}\left(\phi_{\mu}(\mathbf{r}) \phi_{\nu}(\mathbf{r})\right)^{[Q]} \\
& +\sum_{\lambda \sigma} \int \mathrm{d} \mathbf{r} \phi_{\mu}(\mathbf{r}) \phi_{\nu}(\mathbf{r}) \frac{\partial^{2} f_{x c}}{\partial \rho(\mathbf{r})^{2}} P_{\lambda \sigma}\left(\phi_{\lambda}(\mathbf{r}) \phi_{\sigma}(\mathbf{r})\right)^{[Q]} \\
g_{\mu \nu}^{Y[Q]} \equiv & \sum_{\lambda \sigma} P_{\lambda \sigma}^{[Q]} \omega_{\mu \nu \lambda \sigma}
\end{aligned}
$$

Here, the first derivative $\int^{[Q]}$ represents differentiation with respect to the Becke weights in the exchange-correlation numerical quadrature. The total one-electron-integral derivative for TD-DFT can be written as

$$
\begin{aligned}
h_{\mu \nu}^{[Q]} & =h_{\mu \nu}^{0[Q]}+g_{\mu \nu}^{[Q]} \\
& =h_{\mu \nu}^{0[Q]}+\tilde{g}_{\mu \nu}^{[Q]}+g_{\mu \nu}^{Y[Q]} \\
& \equiv \tilde{h}_{\mu \nu}^{[Q]}+g_{\mu \nu}^{Y[Q]}
\end{aligned}
$$

Second, analogous definitions can be made for the twoelectron-integral derivatives $\omega^{[\mathrm{Q}]}$

$$
\omega_{\mu \nu \lambda \sigma}^{[Q]}=\tilde{\omega}_{\mu \nu \lambda \sigma}^{[Q]}+\omega_{\mu \nu \lambda \sigma}^{Y[Q]}
$$

where

$$
\begin{aligned}
\tilde{\omega}_{\mu \nu \lambda \sigma}^{[Q]} \equiv & \int^{[Q]} \mathrm{d} \mathbf{r} \phi_{\mu}(\mathbf{r}) \phi_{\nu}(\mathbf{r}) \frac{\partial^{2} f_{x c}}{\partial \rho(\mathbf{r})^{2}} \phi_{\lambda}(\mathbf{r}) \phi_{\sigma}(\mathbf{r}) \\
& +\int \mathrm{d} \mathbf{r}\left(\phi_{\mu}(\mathbf{r}) \phi_{\nu}(\mathbf{r})\right)^{[Q]} \frac{\partial^{2} f_{x c}}{\partial \rho(\mathbf{r})^{2}} \phi_{\lambda}(\mathbf{r}) \phi_{\sigma}(\mathbf{r}) \\
& +\int \mathrm{d} \mathbf{r} \phi_{\mu}(\mathbf{r}) \phi_{\nu}(\mathbf{r}) \frac{\partial^{2} f_{x c}}{\partial \rho(\mathbf{r})^{2}}\left(\phi_{\lambda}(\mathbf{r}) \phi_{\sigma}(\mathbf{r})\right)^{[Q]} \\
& +\sum_{\gamma \delta} \int \mathrm{d} \mathbf{r} \phi_{\mu}(\mathbf{r}) \phi_{\nu}(\mathbf{r}) \frac{\partial^{3} f_{x c}}{\partial \rho(\mathbf{r})^{3}} \phi_{\lambda}(\mathbf{r}) \phi_{\sigma}(\mathbf{r}) \\
& P_{\gamma \delta}\left(\phi_{\gamma}(\mathbf{r}) \phi_{\delta}(\mathbf{r})\right)^{[Q]} \\
\omega_{\mu \nu \lambda \sigma}^{Y[Q]} \equiv & \sum_{\gamma \delta} P_{\gamma \delta}^{[Q]} \Xi_{\mu \nu \lambda \sigma \gamma \delta}
\end{aligned}
$$

and $\Xi_{\mu \nu \lambda \sigma \gamma \delta}$ is the $x c$ functional third derivative

$$
\Xi_{\mu \nu \lambda \sigma \gamma \delta} \equiv \int \mathrm{d} \mathbf{r} \phi_{\mu}(\mathbf{r}) \phi_{\nu}(\mathbf{r}) \frac{\partial^{3} f_{x c}}{\partial \rho(\mathbf{r})^{3}} \phi_{\lambda}(\mathbf{r}) \phi_{\sigma}(\mathbf{r}) \phi_{\gamma}(\mathbf{r}) \phi_{\delta}(\mathbf{r})
$$


Thus, the total two-electron-integral derivatives for TD-DFT can be written as

$$
\begin{aligned}
\Omega_{\mu \nu \lambda \sigma}^{[Q]} & =\Pi_{\mu \nu \lambda \sigma}^{[Q]}+\omega_{\mu \nu \lambda \sigma}^{[Q]} \\
& =\Pi_{\mu \nu \lambda \sigma}^{[Q]}+\tilde{\omega}_{\mu \nu \lambda \sigma}^{[Q]}+\omega_{\mu \nu \lambda \sigma}^{Y[Q]} \\
& \equiv \tilde{\Omega}_{\mu \nu \lambda \sigma}^{[Q]}+\omega_{\mu \nu \lambda \sigma}^{Y[Q]}
\end{aligned}
$$

With the definitions made above, one can simplify the nonresponse and response terms of the TD-DFT/RPA derivative couplings in a fashion completely analogous to the case in TD-DFT/TDA. ${ }^{40}$ In the end, a complete expression for the TD-DFT derivative coupling is

$$
\begin{aligned}
& \left\langle\Psi_{I} \mid \Psi_{J}^{[Q]}\right\rangle=\frac{1}{E_{J}-E_{I}}\left\{\sum_{\mu \nu} D_{\mu \nu}^{I J} \tilde{h}_{\mu \nu}^{[Q]}\right. \\
& +\sum_{\mu \nu \lambda \sigma}\left[\left(R_{\mu \lambda}^{X I} R_{\sigma \nu}^{X J}+R_{\mu \lambda}^{Y I} R_{\sigma \nu}^{Y J}+R_{\mu \lambda}^{X I} R_{\nu \sigma}^{Y J}+R_{\mu \lambda}^{Y I} R_{\nu \sigma}^{X I}\right)\right. \\
& \left.\times \tilde{\Omega}_{\mu \nu \lambda \sigma}^{[Q]}+D_{\mu \lambda}^{I J} P_{\sigma \nu} \Pi_{\mu \nu \lambda \sigma}^{[Q]}\right] \\
& -\frac{1}{2} \sum_{\alpha \beta \mu \nu} S_{\mu \nu}^{[Q]} \tilde{P}_{\mu \alpha}\left(D_{\beta \nu}^{I J}+D_{\nu \beta}^{I J}\right) F_{\alpha \beta} \\
& -\frac{1}{2} \sum_{\mu \nu \alpha \beta \gamma \delta} S_{\mu \nu}^{[Q]} \tilde{P}_{\mu \alpha}\left(R_{\nu \gamma}^{X I} R_{\delta \beta}^{X J}+R_{\nu \gamma}^{Y I} R_{\delta \beta}^{Y J}+R_{\delta \beta}^{X I} R_{\nu \gamma}^{X J}\right. \\
& \left.+R_{\delta \beta}^{Y I} R_{\nu \gamma}^{Y J}+R_{\nu \gamma}^{X I} R_{\beta \delta}^{Y J}+R_{\nu \gamma}^{Y I} R_{\beta \delta}^{X I}+R_{\beta \delta}^{X I} R_{\nu \gamma}^{Y J}+R_{\beta \delta}^{Y I} R_{\nu \gamma}^{X I}\right) \\
& \times \Omega_{\alpha \beta \gamma \delta}-\frac{1}{2} \sum_{\mu \nu \alpha \beta \gamma \delta} S_{\mu \nu}^{[Q]} \tilde{P}_{\mu \alpha}\left(R_{\gamma \nu}^{X I} R_{\beta \delta}^{X J}+R_{\gamma \nu}^{Y I} R_{\beta \delta}^{Y]}\right. \\
& +R_{\beta \delta}^{X I} R_{\gamma \nu}^{X J}+R_{\beta \delta}^{Y I} R_{\gamma \nu}^{Y J}+R_{\gamma \nu}^{X I} R_{\delta \beta}^{Y J}+R_{\gamma \nu}^{Y I} R_{\delta \beta}^{X I}+R_{\delta \beta}^{X I} R_{\gamma \nu}^{Y J} \\
& \left.+R_{\delta \beta}^{Y \mathrm{YI}} R_{\gamma \nu}^{X]}\right) \Omega_{\alpha \beta \gamma \delta}-\frac{1}{2} \sum_{\mu \nu \alpha \beta \gamma \delta \lambda \sigma} S_{\mu \nu}^{[Q]} \tilde{P}_{\mu \lambda} P_{\nu \sigma} \\
& \times\left(R_{\alpha \gamma}^{X I} R_{\delta \beta}^{X I}+R_{\alpha \gamma}^{Y I} R_{\delta \beta}^{Y J}+R_{\delta \beta}^{X I} R_{\alpha \gamma}^{X I}+R_{\delta \beta}^{Y I} R_{\alpha \gamma}^{Y J}+R_{\alpha \gamma}^{X I} R_{\beta \delta}^{Y I}\right. \\
& \left.+R_{\alpha \gamma}^{Y I} R_{\beta \delta}^{X I}+R_{\beta \delta}^{X I} R_{\alpha \gamma}^{Y J}+R_{\beta \delta}^{Y I} R_{\alpha \gamma}^{X J}\right) \Xi_{\alpha \beta \gamma \delta \lambda \sigma} \\
& +\frac{1}{2} \sum_{\mu \nu \alpha \beta \gamma \delta} S_{\mu \nu}^{[Q]} \tilde{P}_{\mu \alpha} P_{\nu \delta}\left(D_{\gamma \beta}^{I J}+D_{\beta \gamma}^{I I}\right) \Omega_{\alpha \beta \gamma \delta} \\
& \left.-\sum_{b i} L_{b i} \Theta_{b i}^{[Q]}\right\}-\sum_{\mu \nu i a b}\left(X_{i}^{I a} X_{i}^{J b}-Y_{i}^{I a} Y_{i}^{J b}\right) \\
& \times C_{\nu a} C_{\mu b} S_{\mu \nu}^{A[Q]}-\sum_{\mu \nu i j a}\left(X_{i}^{I a} X_{j}^{J a}-Y_{i}^{I a} Y_{j}^{J a}\right) C_{\nu i} C_{\mu j} S_{\mu \nu}^{A[Q]}
\end{aligned}
$$

where

$$
\begin{aligned}
L_{b i}= & \sum_{\mu \nu \lambda \sigma d} C_{\mu b} C_{\lambda d}\left(R_{\nu \sigma}^{X I} X_{i}^{J d}+R_{\nu \sigma}^{Y I} Y_{i}^{J d}+X_{i}^{I d} R_{\nu \sigma}^{X J}+Y_{i}^{I d} R_{\nu \sigma}^{Y J}+R_{\sigma \nu}^{X I} Y_{i}^{J d}\right. \\
& \left.+R_{\sigma \nu}^{Y I} X_{i}^{I d}+X_{i}^{I d} R_{\sigma \nu}^{Y J}+Y_{i}^{I d} R_{\sigma \nu}^{X J}\right) \Omega_{\mu \nu \lambda \sigma} \\
& -\sum_{\mu \nu \lambda \sigma l} C_{\mu l} C_{\lambda i}\left(R_{\nu \sigma}^{X I} X_{l}^{I b}+R_{\nu \sigma}^{Y I} Y_{l}^{I b}+X_{l}^{I b} R_{\nu \sigma}^{X J}+Y_{l}^{I b} R_{\nu \sigma}^{Y J}\right. \\
& \left.+R_{\sigma \nu}^{X I} Y_{l}^{J b}+R_{\sigma \nu}^{Y I} X_{l}^{J b}+X_{l}^{I b} R_{\sigma \nu}^{Y J}+Y_{l}^{I b} R_{\sigma \nu}^{X J}\right) \Omega_{\mu \nu \lambda \sigma} \\
& +\sum_{\mu \nu \lambda \sigma \gamma \delta} C_{\gamma b} C_{\delta i}\left(R_{\mu \lambda}^{X I} R_{\sigma \nu}^{X J}+R_{\mu \lambda}^{Y I} R_{\sigma \nu}^{Y J}+R_{\sigma \nu}^{X I} R_{\mu \lambda}^{X J}+R_{\sigma \nu}^{Y I} R_{\mu \lambda}^{Y J}\right. \\
& \left.+R_{\mu \lambda}^{X I} R_{\nu \sigma}^{Y J}+R_{\mu \lambda}^{Y I} R_{\nu \sigma}^{X I}+R_{\nu \sigma}^{X I} R_{\mu \lambda}^{Y J}+R_{\nu \sigma}^{Y I} R_{\mu \lambda}^{X I}\right) \Xi_{\mu \nu \lambda \sigma \gamma \delta} \\
& +\sum_{\mu \nu \lambda \sigma} C_{\nu b} C_{\sigma i}\left(D_{\mu \lambda}^{I J}+D_{\lambda \mu}^{I J}\right) \Omega_{\mu \nu \lambda \sigma}
\end{aligned}
$$

To evaluate the $\Theta_{b i}^{[Q]}$ terms, one must solve the coupledperturbed Hartree-Fock (CPHF) equation ${ }^{44,45}$ by applying the " $z$-vector" method ${ }^{46}$

$$
z_{a j}=\sum_{b i} L_{b i}\left(\frac{\partial^{2} E}{\partial \Theta_{a j} \partial \Theta_{b i}}\right)^{-1}
$$

Once the $z$-vector has been saved to disk, we construct the mixed derivatives for each coordinate and our final expression becomes

$$
\begin{aligned}
& \left\langle\Psi_{I} \mid \Psi_{J}^{[Q]}\right\rangle=\frac{1}{E_{J}-E_{I}}\left\{\sum_{\mu \nu} \bar{D}_{\mu \nu}^{I J} \tilde{h}_{\mu \nu}^{[Q]}\right. \\
& +\sum_{\mu \nu \lambda \sigma}\left[\left(R_{\mu \lambda}^{X I} R_{\sigma \nu}^{X J}+R_{\mu \lambda}^{Y I} R_{\sigma \nu}^{Y J}+R_{\mu \lambda}^{X I} R_{\nu \sigma}^{Y J}\right.\right. \\
& \left.\left.+R_{\mu \lambda}^{Y I} R_{\nu \sigma}^{X J}\right) \tilde{\Omega}_{\mu \nu \lambda \sigma}^{[Q]}+\bar{D}_{\mu \lambda}^{I J} P_{\sigma \nu} \Pi_{\mu \nu \lambda \sigma}^{[Q]}\right] \\
& -\frac{1}{2} \sum_{\alpha \beta \mu \nu} S_{\mu \nu}^{[Q]} \tilde{P}_{\mu \alpha}\left(\bar{D}_{\beta \nu}^{I J}+\bar{D}_{\nu \beta}^{I J}\right) F_{\alpha \beta} \\
& -\frac{1}{2} \sum_{\mu \nu \alpha \beta \gamma \delta} S_{\mu \nu}^{[Q]} \tilde{P}_{\mu \alpha}\left(R_{\nu \gamma}^{X I} R_{\delta \beta}^{X J}+R_{\nu \gamma}^{Y I} R_{\delta \beta}^{Y J}\right. \\
& +R_{\delta \beta}^{X I} R_{\nu \gamma}^{X J}+R_{\delta \beta}^{Y I} R_{\nu \gamma}^{Y J}+R_{\nu \gamma}^{X I} R_{\beta \delta}^{Y J}+R_{\nu \gamma}^{Y I} R_{\beta \delta}^{X J} \\
& \left.+R_{\beta \delta}^{X I} R_{\nu \gamma}^{Y J}+R_{\beta \delta}^{Y I} R_{\nu \gamma}^{X J}\right) \Omega_{\alpha \beta \gamma \delta} \\
& -\frac{1}{2} \sum_{\mu \nu \alpha \beta \gamma \delta} S_{\mu \nu}^{[Q]} \tilde{P}_{\mu \alpha}\left(R_{\gamma \nu}^{X I} R_{\beta \delta}^{X J}+R_{\gamma \nu}^{Y I} R_{\beta \delta}^{Y J}\right. \\
& +R_{\beta \delta}^{X I} R_{\gamma \nu}^{X J}+R_{\beta \delta}^{Y I} R_{\gamma \nu}^{Y J}+R_{\gamma \nu}^{X I} R_{\delta \beta}^{Y J}+R_{\gamma \nu}^{Y I} R_{\delta \beta}^{X J} \\
& \left.+R_{\delta \beta}^{X I} R_{\gamma \nu}^{Y J}+R_{\delta \beta}^{Y I} R_{\gamma \nu}^{X J}\right) \Omega_{\alpha \beta \gamma \delta} \\
& -\frac{1}{2} \sum_{\mu \nu \alpha \beta \gamma \delta \lambda \sigma} S_{\mu \nu}^{[Q]} \tilde{P}_{\mu \lambda} P_{\nu \sigma}\left(R_{\alpha \gamma}^{X I} R_{\delta \beta}^{X J}+R_{\alpha \gamma}^{Y I} R_{\delta \beta}^{Y J}\right. \\
& +R_{\delta \beta}^{X I} R_{\alpha \gamma}^{X J}+R_{\delta \beta}^{Y I} R_{\alpha \gamma}^{Y J}+R_{\alpha \gamma}^{X I} R_{\beta \delta}^{Y J}+R_{\alpha \gamma}^{Y I} R_{\beta \delta}^{X J} \\
& \left.+R_{\beta \delta}^{X I} R_{\alpha \gamma}^{Y J}+R_{\beta \delta}^{Y I} R_{\alpha \gamma}^{X J}\right) \Xi_{\alpha \beta \gamma \delta \lambda \sigma} \\
& \left.+\frac{1}{2} \sum_{\mu \nu \alpha \beta \gamma \delta} S_{\mu \nu}^{[Q]} \tilde{P}_{\mu \alpha} P_{\nu \delta}\left(\bar{D}_{\gamma \beta}^{I J}+\bar{D}_{\beta \gamma}^{I J}\right) \Omega_{\alpha \beta \gamma \delta}\right\} \\
& -\sum_{\mu \nu i a b}\left(X_{i}^{I a} X_{i}^{J b}-Y_{i}^{I a} Y_{i}^{J b}\right) C_{\nu a} C_{\mu b} S_{\mu \nu}^{A[Q]} \\
& -\sum_{\mu \nu i j a}\left(X_{i}^{I a} X_{j}^{J a}-Y_{i}^{I a} Y_{j}^{J a}\right) C_{\nu i} C_{\mu j} S_{\mu \nu}^{A[Q]}
\end{aligned}
$$

where $\bar{D}^{I J}$ represents the relaxed difference density matrix

$$
\begin{aligned}
\bar{D}_{\mu \nu}^{I J} & \equiv D_{\mu \nu}^{I J}-\sum_{a j} z_{a j}\left(C_{\mu a} C_{\nu j}+C_{\mu j} C_{\nu a}\right) \\
& =D_{\mu \nu}^{I J}-\left(z_{\mu \nu}+z_{\nu \mu}\right)
\end{aligned}
$$

Before ending, we note that this formal expression (eq 50) can be easily transformed to the energy gradient when setting $I=J$. Also, eq 50 can be derived from a Hellmann-Feynman approach (just as for TD-HF in ref 41). See Appendix A.

\section{COMPARISON WITH FINITE-DIFFERENCE}

In order to check our implementation of eqs 50 and 51 for TD-DFT/RPA derivative couplings, in Table 1 , we compare the $S_{1} / S_{5}$ derivative coupling of formaldehyde calculated directly from eq 50 with the finite-difference data given by the formula below 
Table 1. Derivative Couplings (in $a_{0}^{-1}$ ) between the $S_{1}$ and $S_{5}$ States of Formaldehyde (HCHO) as Computed by FiniteDifference (FD) and Analytical Theory (DC) ${ }^{a}$

\begin{tabular}{|c|c|c|c|c|c|c|}
\hline \multirow[b]{2}{*}{ degree of freedom $(Q)$} & \multicolumn{2}{|c|}{ B3LYP } & \multicolumn{2}{|c|}{$\omega \mathrm{B} 97$} & \multicolumn{2}{|c|}{$\omega \mathrm{B} 97 \mathrm{X}$} \\
\hline & FD & $\mathrm{DC}$ & FD & $\mathrm{DC}$ & FD & $\mathrm{DC}$ \\
\hline $\mathrm{C}_{y}$ & -0.88668 & -0.88669 & -0.84566 & -0.84567 & -0.85341 & -0.85337 \\
\hline $\mathrm{H}_{x}$ & -0.05466 & -0.05470 & -0.04737 & -0.04739 & -0.04945 & -0.04946 \\
\hline $\mathrm{H}_{y}$ & 0.10099 & 0.10099 & 0.09228 & 0.09224 & 0.09432 & 0.09433 \\
\hline $\mathrm{O}_{y}$ & 0.41617 & 0.41620 & 0.39715 & 0.39715 & 0.39984 & 0.39989 \\
\hline
\end{tabular}

${ }^{a}$ Note that only the components larger than $10^{-4} a_{0}^{-1}$ are listed here.

$$
\begin{aligned}
& \left\langle\Psi_{I} \mid \Psi_{J}^{[Q]}\right\rangle \approx \\
& \frac{\left\langle\Psi_{I}(Q) \mid \Psi_{J}(Q+\Delta Q)\right\rangle-\left\langle\Psi_{I}(Q) \mid \Psi_{J}(Q-\Delta Q)\right\rangle}{2 \Delta Q}
\end{aligned}
$$

where $Q$ indexes the nuclear degree of freedom. To evaluate the overlaps in eq 52, we employ the pseudo-wavefunction ansatz in ref 41 and calculate the finite-difference results according to

$$
\begin{aligned}
\left\langle\Psi_{I} \mid \Psi_{J}^{[Q]}\right\rangle \approx & \frac{\langle X(Q) \mid X(Q+\Delta Q)\rangle-\langle X(Q) \mid X(Q-\Delta Q)\rangle}{2 \Delta Q} \\
& -\frac{\langle y(Q) \mid y(Q+\Delta Q)\rangle-\langle y(Q) \mid y(Q-\Delta Q)\rangle}{2 \Delta Q}
\end{aligned}
$$

where we define

$$
\begin{aligned}
& x \equiv \sum_{i a} X_{i}^{I a} \hat{a}_{a}^{\dagger} \hat{a}_{i}\left|\Phi_{\mathrm{DFT}}\right\rangle \\
& y \equiv \sum_{i a} Y_{i}^{I a} \hat{a}_{a}^{\dagger} \hat{a}_{i}\left|\Phi_{\mathrm{DFT}}\right\rangle
\end{aligned}
$$

The quantum chemistry package Q-Chem ${ }^{47,48}$ was used for all calculations. Three TD-DFT $x c$ functionals (B3LYP, ${ }^{49,50}$ $\omega \mathrm{B} 97$, and $\left.\omega \mathrm{B} 97 \mathrm{X}^{51}\right)$ were tested using the $6-31 \mathrm{G}^{*}$ basis set. As shown in Table 1, our numerical results from eq 50 match the finite-difference data with an error less than $10^{-4}$ for each $x c$ functional, which validates our implementation of the TD-DFT/RPA derivative couplings.

\section{GEOMETRIC PHASE AND BRANCHING PLANE FOR RPA CONICAL INTERSECTIONS}

The derivative couplings calculated above in eq 50 were derived under several assumptions, especially the necessity of small $\mathbf{Y}$ terms, which allowed us to ignore and/or manipulate several terms in the derivation. To further justify our theory, we will now evaluate our derivative couplings around a CI and examine whether or not we recover the correct properties, which is an essential criterion for any derivative coupling approach. In particular, for the exact eigenstates of the Hamiltonian, the derivative couplings must satisfy two criteria around a CI: (i) the derivative couplings must be in the branching plane; (ii) the derivative couplings must obey Berry's phase.

To date, almost all examinations of Berry's phase and branching planes have focused on $2 \times 2$ Hermitian Hamiltonians. ${ }^{26,27}$ In our case, there are two slight complications because (i) the Hamiltonian is not Hermitian and (ii) the Hamiltonian eigenstates come in pairs of positive and negative energy.

Thus, whereas standard quantum chemistry considers a $2 \times 2$ Hermitian Hamiltonian for two diabatic states, we must now consider a $4 \times 4$ non-Hermitian Hamiltonian with four diabatic states. In particular, for TD-DFT/RPA, the non-Hermitian Hamiltonian

$$
\mathbf{H}=\left(\begin{array}{ll}
\mathbf{A} & \mathbf{B} \\
-\mathbf{B} & -\mathbf{A}
\end{array}\right)
$$

around a CI can be written as (to the first order)

$$
\mathbf{H}(\mathbf{R})=\left(\begin{array}{llll}
\varepsilon-\mathbf{g} \cdot \mathbf{R} & \mathbf{h} \cdot \mathbf{R} & \mathbf{b}_{11} \cdot \mathbf{R} & \mathbf{b}_{12} \cdot \mathbf{R} \\
\mathbf{h} \cdot \mathbf{R} & \varepsilon+\mathbf{g} \cdot \mathbf{R} & \mathbf{b}_{21} \cdot \mathbf{R} & \mathbf{b}_{22} \cdot \mathbf{R} \\
-\mathbf{b}_{11} \cdot \mathbf{R} & -\mathbf{b}_{12} \cdot \mathbf{R} & -\varepsilon+\mathbf{g} \cdot \mathbf{R} & -\mathbf{h} \cdot \mathbf{R} \\
-\mathbf{b}_{21} \cdot \mathbf{R} & -\mathbf{b}_{22} \cdot \mathbf{R} & -\mathbf{h} \cdot \mathbf{R} & -\varepsilon-\mathbf{g} \cdot \mathbf{R}
\end{array}\right)
$$

Let the basis here be denoted as $\left\{\left|\Psi_{I}^{0}\right\rangle\right\}$, where $I=1,2,3,4$. In this basis, $\mathbf{g}$ and $\mathbf{h}$ are the matrix elements of $\mathbf{A}$ around the CI point, $\varepsilon$ is the (positive) energy of two TD-DFT/RPA states at the CI point, and $\mathbf{b}_{i j}$ is a matrix representation of $\mathbf{B}$. The Hamiltonian has been expanded to first order in $\mathbf{R}$.

We will now perform a series of linear transformations. Our first step is to propose a branching plane. We propose that the branching plane should be spanned by the $\mathbf{g}$ and $\mathbf{h}$ vectors. Let $\theta$ be the branching angle, $\theta=0^{\circ}$, in the $\mathrm{g}$ direction. Second, we rescale $\mathbf{g}$ and $\mathbf{h}$ to make the norm of the gradient difference indistinguishable at every direction around the $\mathrm{CI}$ by taking $g=\rho \cos \theta$ and $h=\rho \sin \theta$. Third, the $\mathbf{A}$ and $\mathbf{- A}$ matrix blocks can be diagonalized everywhere in the branching plane and the Hamiltonian becomes

$$
\begin{aligned}
\mathbf{H}\left(\rho, \theta, R^{\perp}\right) & =\left(\begin{array}{llll}
\varepsilon-\rho & 0 & \tilde{b}_{11}\left(\rho, \theta, R^{\perp}\right) & \tilde{b}_{12}\left(\rho, \theta, R^{\perp}\right) \\
0 & \varepsilon+\rho & \tilde{b}_{21}\left(\rho, \theta, R^{\perp}\right) & \tilde{b}_{22}\left(\rho, \theta, R^{\perp}\right) \\
-\tilde{b}_{11}\left(\rho, \theta, R^{\perp}\right) & -\tilde{b}_{12}\left(\rho, \theta, R^{\perp}\right) & -\varepsilon+\rho & 0 \\
-\tilde{b}_{21}\left(\rho, \theta, R^{\perp}\right) & -\tilde{b}_{22}\left(\rho, \theta, R^{\perp}\right) & 0 & -\varepsilon-\rho
\end{array}\right) \\
& \equiv \mathbf{H}_{0}(\rho)+\tilde{\mathbf{B}}\left(\rho, \theta, R^{\perp}\right)
\end{aligned}
$$


where $R^{\perp}$ denotes the coordinate perpendicular to the branching plane. By doing the linear transformation, $\tilde{b}_{i j}$ linearly depends on $\rho$ and/or $R^{\perp}$ and can be decomposed into two components: $\widetilde{b}_{i j}=\boldsymbol{\rho} \cdot \mathbf{b}_{i j}^{0}+\mathbf{R}^{\perp} \cdot \mathbf{b}_{i j}^{\perp}$. Here $\mathbf{b}_{i j}^{0}$ lies in the branching plane and $\mathbf{b}_{i j}^{\perp}$ is perpendicular to the branching plane. In eq $59, \mathbf{H}_{0}$ contains only the diagonal elements (eq 60) and $\tilde{\mathbf{B}}$ contains only the off-diagonal elements of $\mathbf{H}$ (eq 61).

$$
\mathbf{H}_{0}(\rho)=\left(\begin{array}{llll}
\varepsilon-\rho & 0 & 0 & 0 \\
0 & \varepsilon+\rho & 0 & 0 \\
0 & 0 & -\varepsilon+\rho & 0 \\
0 & 0 & 0 & -\varepsilon-\rho
\end{array}\right)
$$

$\tilde{\mathbf{B}}\left(\rho, \theta, R^{\perp}\right)=$

$$
\left(\begin{array}{llll}
0 & 0 & \tilde{b}_{11}\left(\rho, \theta, R^{\perp}\right) & \tilde{b}_{12}\left(\rho, \theta, R^{\perp}\right) \\
0 & 0 & \tilde{b}_{21}\left(\rho, \theta, R^{\perp}\right) & \tilde{b}_{22}\left(\rho, \theta, R^{\perp}\right) \\
-\tilde{b}_{11}\left(\rho, \theta, R^{\perp}\right) & -\tilde{b}_{12}\left(\rho, \theta, R^{\perp}\right) & 0 & 0 \\
-\tilde{b}_{21}\left(\rho, \theta, R^{\perp}\right) & -\tilde{b}_{22}\left(\rho, \theta, R^{\perp}\right) & 0 & 0
\end{array}\right)
$$

Note that the basis $\left\{\left|\tilde{\Psi}_{I}^{0}\right\rangle\right\}$ for eqs $58-61$ depends on $\theta$ :

$$
\begin{aligned}
& \left|\tilde{\Psi}_{1}^{0}(\theta)\right\rangle=\cos \frac{\theta}{2}\left(\begin{array}{l}
1 \\
0 \\
0 \\
0
\end{array}\right)+\sin \frac{\theta}{2}\left(\begin{array}{l}
0 \\
1 \\
0 \\
0
\end{array}\right) \\
& \left|\tilde{\Psi}_{2}^{0}(\theta)\right\rangle=\cos \frac{\theta}{2}\left(\begin{array}{l}
0 \\
1 \\
0 \\
0
\end{array}\right)-\sin \frac{\theta}{2}\left(\begin{array}{l}
1 \\
0 \\
0 \\
0
\end{array}\right) \\
& \left|\tilde{\Psi}_{3}^{0}(\theta)\right\rangle=\cos \frac{\theta}{2}\left(\begin{array}{l}
0 \\
0 \\
1 \\
0
\end{array}\right)+\sin \frac{\theta}{2}\left(\begin{array}{l}
0 \\
0 \\
0 \\
1
\end{array}\right) \\
& \left|\tilde{\Psi}_{4}^{0}(\theta)\right\rangle=\cos \frac{\theta}{2}\left(\begin{array}{l}
0 \\
0 \\
0 \\
1
\end{array}\right)-\sin \frac{\theta}{2}\left(\begin{array}{l}
0 \\
0 \\
1 \\
0
\end{array}\right)
\end{aligned}
$$

It is crucial to keep in mind that, in changing coordinates, we have performed only linear transformations.

Because $\mathbf{H}$ is antisymmetric, we will need to construct the "left" and "right" eigenfunctions, $\left\langle\Psi_{I}^{\mathrm{L}}\right|$ and $\left\langle\Psi_{I}^{\mathrm{R}}\right|$, of eq 56 :

$$
\left|\Psi_{I}^{\mathrm{L}}\right\rangle \equiv\left(\begin{array}{ll}
\mathbf{X}^{I} & -\mathbf{Y}^{I}
\end{array}\right), \quad\left|\Psi_{I}^{\mathrm{R}}\right\rangle \equiv\left(\begin{array}{c}
\mathbf{X}^{I} \\
\mathbf{Y}^{I}
\end{array}\right)
$$

where

$$
\begin{aligned}
& \left\langle\Psi_{I}^{\mathrm{L}}\right| \mathbf{H}=E_{I}\left\langle\Psi_{I}^{\mathrm{L}}\right| \\
& \mathbf{H}\left|\Psi_{I}^{\mathrm{R}}\right\rangle=E_{I}\left|\Psi_{I}^{\mathrm{R}}\right\rangle
\end{aligned}
$$

We now assume that the elements of $\mathbf{B}$ (or $\tilde{\mathbf{B}}$ ) are small compared with the diagonal elements of $\mathbf{A}$-this is consistent with the assumption that RPA is most accurate, the magnitude of $\mathbf{Y}$ should be small. ${ }^{8}$ In particular, we assume that $\tilde{b}_{i j} / \varepsilon \ll 1$.
Thus, we can estimate the energies (i.e., eigenvalues) of $\mathbf{H}$. Using second order perturbation theory

$$
\begin{aligned}
E_{1} & =\varepsilon-\rho+\frac{\left|\left\langle\tilde{\Psi}_{3}^{0}|\tilde{\mathbf{B}}| \tilde{\Psi}_{1}^{0}\right\rangle\right|^{2}}{2(\varepsilon-\rho)}+\frac{\left|\left\langle\tilde{\Psi}_{4}^{0}|\tilde{\mathbf{B}}| \tilde{\Psi}_{1}^{0}\right\rangle\right|^{2}}{2 \varepsilon} \\
& =\varepsilon-\rho+O\left(\tilde{\mathbf{B}}^{2}\right) \\
E_{2} & =\varepsilon+\rho+\frac{\left|\left\langle\tilde{\Psi}_{3}^{0}|\tilde{\mathbf{B}}| \tilde{\Psi}_{2}^{0}\right\rangle\right|^{2}}{2 \varepsilon}+\frac{\left|\left\langle\tilde{\Psi}_{4}^{0}|\tilde{\mathbf{B}}| \tilde{\Psi}_{2}^{0}\right\rangle\right|^{2}}{2(\varepsilon+\rho)} \\
& =\varepsilon+\rho+O\left(\tilde{\mathbf{B}}^{2}\right)
\end{aligned}
$$

From this, we may conclude that the eigenvalues of $\mathbf{H}$ are the same as the eigenvalues of $\mathrm{H}_{0}$ through $O\left(\rho^{2}\right), O\left(\rho R^{\perp}\right)$, or $O\left(R^{\perp^{2}}\right)$ (since $\tilde{\mathbf{B}}$ linearly depends on $\rho$ and/or $R^{\perp}$ ) and thus the $\mathbf{g}-\mathbf{h}$ plane is indeed the correct branching plane.

Next, our second task is to consider the derivative couplings. We are interested in the first two eigenstates with positive energy; these pseudo-wavefunctions can be expressed as (to the first order)

$$
\begin{aligned}
& \left|\Psi_{1}^{\mathrm{R}}\right\rangle=\left|\tilde{\Psi}_{1}^{0}\right\rangle+\frac{\left\langle\tilde{\Psi}_{3}^{0}\left|\tilde{\mathbf{B}}_{1}\right| \tilde{\Psi}_{1}^{0}\right\rangle}{2(\varepsilon-\rho)}\left|\tilde{\Psi}_{3}^{0}\right\rangle+\frac{\left\langle\tilde{\Psi}_{4}^{0}\left|\tilde{\mathbf{B}}^{\prime}\right| \tilde{\Psi}_{1}^{0}\right\rangle}{2 \varepsilon}\left|\tilde{\Psi}_{4}^{0}\right\rangle \\
& \left|\Psi_{2}^{\mathrm{R}}\right\rangle=\left|\tilde{\Psi}_{2}^{0}\right\rangle+\frac{\left\langle\tilde{\Psi}_{3}^{0}\left|\tilde{\mathbf{B}}_{2}\right| \tilde{\Psi}_{2}^{0}\right\rangle}{2 \varepsilon}\left|\tilde{\Psi}_{3}^{0}\right\rangle+\frac{\left\langle\tilde{\Psi}_{4}^{0}|\tilde{\mathbf{B}}| \tilde{\Psi}_{2}^{0}\right\rangle}{2(\varepsilon+\rho)}\left|\tilde{\Psi}_{4}^{0}\right\rangle \\
& \left\langle\Psi_{1}^{\mathrm{L}}\right|=\left\langle\tilde{\Psi}_{1}^{0}\right|-\left\langle\tilde{\Psi}_{3}^{0}\right| \frac{\left\langle\tilde{\Psi}_{3}^{0}|\tilde{\mathbf{B}}| \tilde{\Psi}_{1}^{0}\right\rangle}{2(\varepsilon-\rho)}-\left\langle\tilde{\Psi}_{4}^{0}\right| \frac{\left\langle\tilde{\Psi}_{4}^{0}|\tilde{\mathbf{B}}| \tilde{\Psi}_{1}^{0}\right\rangle}{2 \varepsilon} \\
& \left\langle\Psi_{2}^{\mathrm{L}}\right|=\left\langle\tilde{\Psi}_{2}^{0}\right|-\left\langle\tilde{\Psi}_{3}^{0}\right| \frac{\left\langle\tilde{\Psi}_{3}^{0}|\tilde{\mathbf{B}}| \tilde{\Psi}_{2}^{0}\right\rangle}{2 \varepsilon}-\left\langle\tilde{\Psi}_{4}^{0}\right| \frac{\left\langle\tilde{\Psi}_{4}^{0}|\tilde{\mathbf{B}}| \tilde{\Psi}_{2}^{0}\right\rangle}{2(\varepsilon+\rho)}
\end{aligned}
$$

where $\left\{\left|\tilde{\Psi}_{I}^{0}\right\rangle\right\}$ are the eigenstates of $\mathbf{H}_{0}$. Because $\left\{\left|\tilde{\Psi}_{I}^{0}\right\rangle\right\}$ depend only on $\theta$, it is clear that our derivative couplings lie in the branching plane.

Finally, our third task is to compute the exact direction of the derivative couplings. The derivative coupling between two RPA states is

$$
\left\langle\Psi_{1}^{\mathrm{L}} \mid \frac{\partial}{\partial Q} \Psi_{2}^{\mathrm{R}}\right\rangle=\left\langle\tilde{\Psi}_{1}^{0} \mid \frac{\partial}{\partial Q} \tilde{\Psi}_{2}^{0}\right\rangle+O(\tilde{\mathbf{B}})+O\left(\tilde{\mathbf{B}}^{2}\right)
$$

where the latter two terms are the couplings contributed by the $\tilde{\mathbf{B}}$ matrix. Note that the $O(\tilde{\mathbf{B}})$ and $O\left(\tilde{\mathbf{B}}^{2}\right)$ terms become infinitesimal in the vicinity of a CI point so that only the first term in eq 77 gives a nonzero contribution to the derivative couplings around a CI.

$$
\left\langle\Psi_{1}^{\mathrm{L}} \mid \frac{\partial}{\partial Q} \Psi_{2}^{\mathrm{R}}\right\rangle \approx\left\langle\tilde{\Psi}_{1}^{0} \mid \frac{\partial}{\partial Q} \tilde{\Psi}_{2}^{0}\right\rangle
$$

From eqs 62,63 , and 78, we may finally conclude that, just as for CIS and TD-DFT/TDA, we can expect standard Berry phase behavior between two RPA states for a loop around the CI:

$$
\phi=\oint_{C}\left\langle\Psi_{1}^{\mathrm{L}} \mid \frac{\partial}{\partial Q} \Psi_{2}^{\mathrm{R}}\right\rangle \mathrm{d} Q \approx \oint_{C}\left\langle\tilde{\Psi}_{1}^{0} \mid \frac{\partial}{\partial Q} \tilde{\Psi}_{2}^{0}\right\rangle \mathrm{d} Q=\pi
$$

We now want to emphasize that our approach for derivative couplings (in eq 18) must have the correct behavior around CIs. After all, we compute derivative couplings by looking at 
the direct overlap of $\left\langle X_{i}^{I a} \Phi_{i}^{a[Q]},-Y_{i}^{I a} \Phi_{i}^{a[Q]}\right|$ with $\mid X_{j}^{J b} \Phi_{i}^{b[Q]}$, $\left.Y_{j}^{b b} \Phi_{j}^{b[Q]}\right\rangle$. See, for instance, the finite-difference data in Table 1 . Though there may be other approaches for derivative couplings that recover the correct physics around a CI, direct differentiation is always guaranteed to work. Thus, given that the TD-HF or TD-DFT/RPA derivative couplings should satisfy a topology and Berry's phase constraint around a CI, eq 79 is an excellent criterion by which we can judge the reasonability of TD-HF or TD-DFT/RPA derivative couplings.

\section{APPLICATION TO PROTONATED FORMALDIMINE}

To convince the reader that our approach does recover the correct behavior around a $\mathrm{CI}$ in practice, we now provide a numerical example: we study the $S_{1} / S_{2}$ CI for protonated formaldimine $\left(\mathrm{CH}_{2} \mathrm{NH}_{2}^{+}\right)$, as previously examined by Tavernelli and co-workers. ${ }^{34-37}$ The minimum crossing point between $S_{1}$ and $\mathrm{S}_{2}$ excited singlet states was determined using the penalty function approach of Martinez et al. ${ }^{33}$ We consider both TD-HF and TD-DFT/RPA excited states and calculate their respective $S_{1} / S_{2}$ derivative coupling around the CI. All the calculations were carried out with the $6-31 \mathrm{G}^{* *}$ basis set.

After the minimum crossing point was located, the branching plane of the $\mathrm{S}_{1} / \mathrm{S}_{2} \mathrm{CI}$ of $\mathrm{CH}_{2} \mathrm{NH}_{2}{ }^{+}$was obtained with the approach described in ref 52 . The $\mathbf{g}$ and $\mathbf{h}$ vectors are defined as follows: (i) define $\vec{D}(\theta)$ as the energy gradient difference in the branching plane at angle $\theta$ around the CI point; (ii) $\mathbf{g}$ is defined as $\vec{D}\left(\theta_{\max }\right)$ when $\theta_{\max }$ is the branching angle that maximizes $\|\vec{D}(\theta)\|$; (iii) $\mathbf{h}$ is defined as the gradient difference with the minimum norm, $\vec{D}\left(\theta_{\text {min }}\right)$. A pair of rescaled vectors, $\mathbf{x}$ and $\mathbf{y}$, is then generated to be used as the coordinates for a loop on the branching plane (with the norm of the gradient difference identical in every direction):

$$
\begin{aligned}
& \mathbf{x}=\frac{1}{\|\mathbf{g}\|} \mathbf{g} \\
& \mathbf{y}=\frac{\|\mathbf{g}\|}{\|\mathbf{h}\|^{2}} \mathbf{h}
\end{aligned}
$$

A total of 36 geometries around the CI point were chosen at the distance $r=0.001 \AA^{53}$ to perform the derivative coupling calculation:

$$
\begin{gathered}
\mathbf{R}(\theta)=\mathbf{R}_{\mathrm{CI}}+0.001(\mathbf{x} \cos \theta+\mathbf{y} \sin \theta), \\
\theta=0^{\circ}, 10^{\circ}, 20^{\circ}, \ldots, 350^{\circ}
\end{gathered}
$$

where $\mathbf{R}_{\mathrm{CI}}$ is the coordinate of the CI point. For a detailed explanation of the TD-HF derivative couplings, see ref 41 . The $\omega \mathrm{B} 97 \mathrm{X}$ functional was used for our TD-DFT/RPA calculations. Previous studies by Tretiak and co-workers have shown the significant contribution of orbital response for semiempirical excited state-excited state derivative couplings. ${ }^{54}$ In order to assess the importance of such orbital response terms $\left(\boldsymbol{\Theta}^{[Q]}\right)$ for RPA derivative couplings in this work, we computed the TD-HF and TD-DFT/RPA derivative couplings with and without response:

(1) Full-DC: complete derivative couplings given by eq 50 .

(2) NR-DC: derivative couplings given by eq 47 without the $\boldsymbol{\Theta}^{[Q]}$ part.

A. Results. In Figure 1, we plot the derivative coupling vectors for each point on the loop around the CI. As Figure 1 shows, for both TD-HF and TD-DFT/RPA, the full-DC vectors

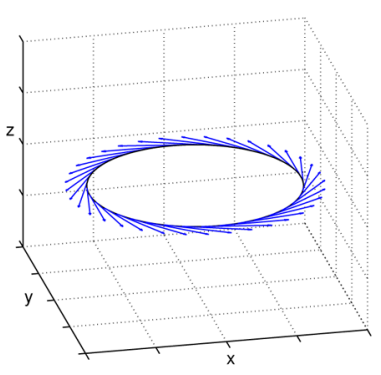

(a) TD-HF full-DC

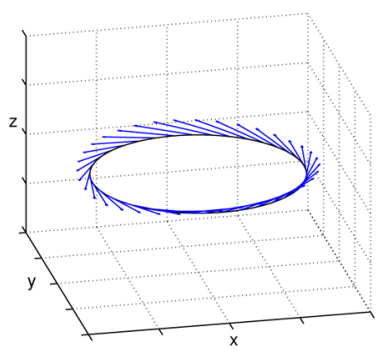

(c) TD-HF NR-DC

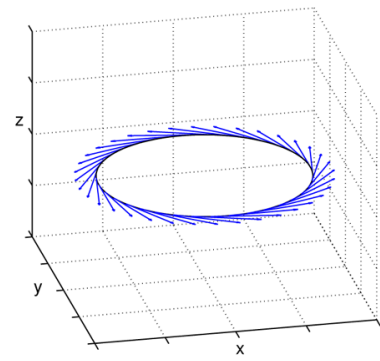

(b) TD-DFT full-DC

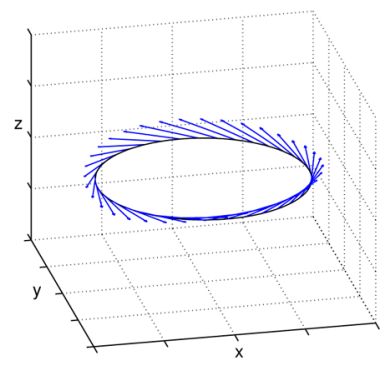

(d) TD-DFT NR-DC
Figure 1. $\mathrm{CH}_{2} \mathrm{NH}_{2}{ }^{+} \mathrm{S}_{1} / \mathrm{S}_{2}$ derivative coupling vectors on the circular loop $(r=0.001 \AA)$ around the CI point in the branching plane given by TD-HF (left) and TD-DFT (right). 36 single-point calculations were performed.

lie rigorously on the branching plane for all geometries computed. By contrast, the NR-DC vectors do not reproduce the correct branching plane. The out-of-plane angle varies from 1 to $15^{\circ}$ for both TD-HF and TD-DFT/RPA. In Figure 2, we

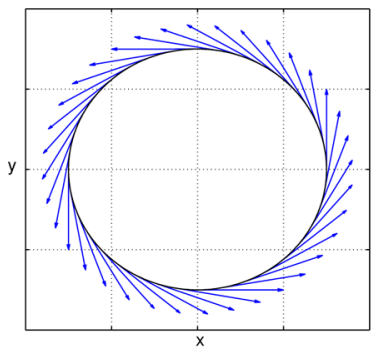

(a) TD-HF full-DC

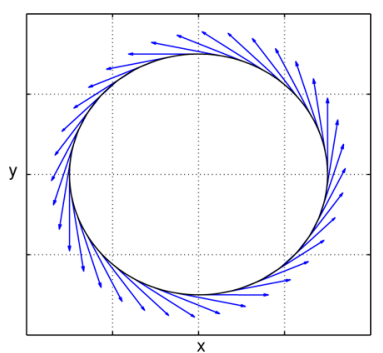

(c) TD-HF NR-DC

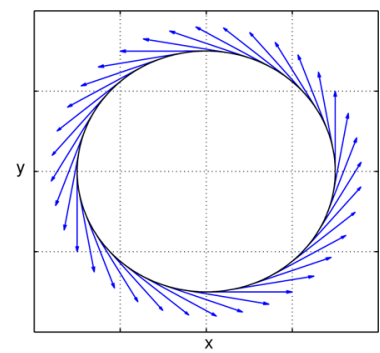

(b) TD-DFT full-DC

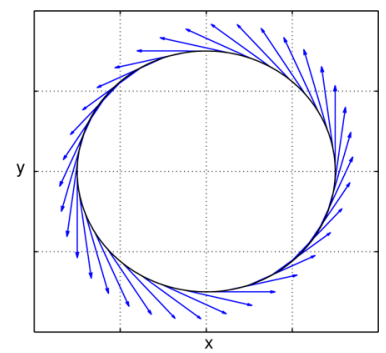

(d) TD-DFT NR-DC
Figure 2. Projected derivative coupling vectors on the circular loop $(r=0.001 \AA)$ around the CI point in the branching plane given by TD-HF (left) and TD-DFT (right).

project the derivative coupling vectors onto the branching plane to get a clearer description of their behavior around the CI point. As can be seen from Figure 2, the full-DC vectors are perpendicular to the gradient difference direction and their 
magnitudes are identical for each point on the loop. The NRDC vectors, however, do not always point to the tangent direction, nor do they show the same magnitude for different points. The results indicate the overwhelming necessity of taking the orbital response terms into consideration.

Using $\theta=90^{\circ}$ as an example, we visualize the full-DC derivative coupling vectors and the gradient difference vectors for both methods in Figure 3. While the gradient difference vectors

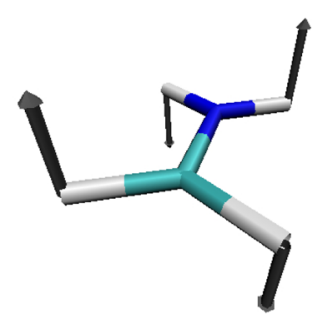

(a) TD-HF gradient difference

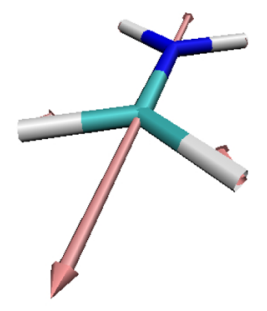

(c) TD-HF derivative coupling

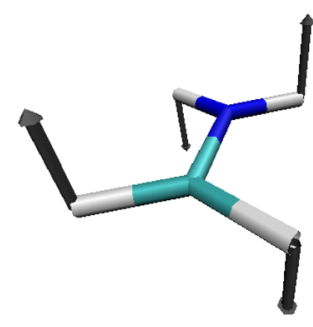

(b)TD-DFT gradient difference

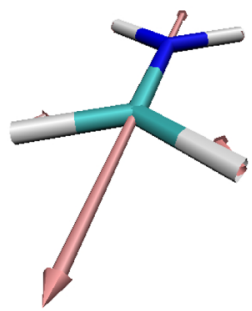

(d) TD-DFT derivative coupling
Figure 3. Derivative coupling and gradient difference vectors for protonated formaldimine at $\theta=90^{\circ}$ given by TD-HF (left) and TDDFT (right).

at the given geometry point perpendicularly to the molecular $\left(\mathrm{CH}_{2} \mathrm{NH}_{2}{ }^{+}\right)$plane, the derivative coupling vectors lie rigorously in the $\left(\mathrm{CH}_{2} \mathrm{NH}_{2}^{+}\right)$plane.

Lastly, as demonstrated in section IV, it is important to check the path integral of the derivative coupling around the CI point to see if it gives the correct Berry phase. The path integral of the derivative coupling vectors is given by

$$
\phi=\oint_{C} \mathbf{d}_{I J}(\mathbf{R}) \cdot \mathrm{d} \mathbf{R}=\int_{0}^{2 \pi} r \mathbf{d}_{I J}(\boldsymbol{\theta}) \cdot \mathrm{d} \boldsymbol{\theta} \approx \sum_{i=1}^{36} r \mathbf{d}_{I J}\left(\boldsymbol{\theta}_{i}\right) \cdot \delta \boldsymbol{\theta}_{i}
$$

As shown in Table 2, both TD-HF and TD-DFT/RPA full-DC vectors reproduced the expected Berry phase perfectly.

Table 2. Circulations of Derivative Coupling Vectors around the $S_{1} / S_{2}$ CI Point of Protonated Formaldimine ${ }^{a}$

\begin{tabular}{lll} 
& \multicolumn{2}{c}{ magnitude (in units of $\pi$ ) } \\
\cline { 2 - 3 } \multicolumn{1}{c}{ terms } & TD-HF & TD-DFT \\
full-DC & 0.99975 & 0.99984 \\
NR-DC (projected) & 0.88373 & 0.93946
\end{tabular}

${ }^{a}$ Note that only the full-DC recovers the correct Berry phase for both TD-HF and TD-DFT.

The projected NR-DC vectors do not satisfy eq 83 (though the error is not enormous). Overall, our calculations highlight the reasonability of our analytic gradient theory for RPA derivative couplings as well as the non-negligible role played by the orbital response terms.

\section{THE CHERNYAK-MUKAMEL EXPRESSION AND THE TRANSITION DENSITY MATRIX ACCORDING TO RESPONSE THEORY}

Before ending, we emphasize that the theoretical approach in this paper (leading up to eq 18) is consistent with the ChernyakMukamel (CM) equality near a crossing. The CM equality that the derivative couplings should take the form

$$
\mathbf{d}_{I J}^{\mathrm{CM}}=\frac{1}{E_{J}-E_{I}} \sum_{p q} \nu_{p q}^{[Q]} \gamma_{p q}^{I J}
$$

where $\gamma_{p q}^{I J}$ is the one-electron transition density matrix. Indeed, for the case of TD-HF, ref 41 shows that our derivative coupling can be put in the form

$$
\mathbf{d}_{I J}^{\mathrm{CB}}=\frac{1}{E_{J}-E_{I}} \sum_{p q} v_{p q}^{[Q]} \Gamma_{p q}^{I J}
$$

where $\Gamma_{p q}^{I J}$ is given by

$$
\begin{aligned}
& \Gamma_{p q}^{I J}= \\
& \left\{\begin{array}{lc}
-\sum_{a}\left(X_{p}^{J a} X_{q}^{I a}+Y_{p}^{I a} Y_{q}^{J a}\right) & \text { for } p, q \in \text { occupied } \\
\sum_{i}\left(X_{i}^{I p} X_{i}^{J q}+Y_{i}^{J p} Y_{i}^{I q}\right) & \text { for } p, q \in \text { virtual orbitals } \\
-\frac{1}{2} \sum_{b i}(A+B)_{q p i b}^{-1} L_{b i} & \text { for } p \in \text { virtual orbitals, } \\
-\frac{1}{2} \sum_{b i}(A+B)_{p q i b}^{-1} L_{b i} & \text { for } q \in \text { occupied orbitals } \\
& p \in \text { occupied orbitals }
\end{array}\right.
\end{aligned}
$$

and $\mathbf{L}$ is the Lagrangian for TD-HF orbital response. Near a crossing, ref 41 shows that $\Gamma_{i j}^{I J}=\gamma_{i j}^{I J}$ and $\Gamma_{a j}^{I J}+\Gamma_{j a}^{I J}=\gamma_{a j}^{(1), I J}+\gamma_{j a}^{(2), I J}$, provided that the transition density matrix $\left(\gamma^{I J}\right)$ is computed with time-dependent response theory.

For the case of TD-DFT/RPA, one finds that the same correspondence between our analytic derivative couplings and the CM expression holds. The only difference between the case of TD-HF and the case of TD-DFT is the fact that our Lagrangian is now the TD-DFT Lagrangian. In particular, for the case of TD-DFT, the $\gamma_{a j}^{(1), I J}$ and $\gamma_{j a}^{(2), I J}$ satisfy

$$
\left[\left(\begin{array}{ll}
\mathbf{A} & \mathbf{B} \\
\mathbf{B} & \mathbf{A}
\end{array}\right)+\Delta E\left(\begin{array}{cc}
\mathbf{I} & 0 \\
0 & -\mathbf{I}
\end{array}\right)\right]\left(\begin{array}{l}
\gamma^{(1), I J} \\
\gamma^{(2), I J}
\end{array}\right)=-\left(\begin{array}{l}
\mathbf{L}^{(1)} \\
\mathbf{L}^{(2)}
\end{array}\right)
$$

where

$$
\begin{aligned}
& L_{b i}^{(1)}=\sum_{p q} \Omega_{p b q i} D_{p q}^{I J}+\sum_{j c d}\left[\left(X_{i}^{I d} X_{j}^{J c}+Y_{j}^{I c} Y_{i}^{J d}\right) \Omega_{c b j d}\right. \\
& \left.+\left(X_{i}^{I d} Y_{i}^{J c}+X_{j}^{I c} Y_{i}^{J d}\right) \Omega_{c d j b}\right]-\sum_{j l c}\left[\left(X_{l}^{I b} X_{j}^{J c}+Y_{j}^{I c} Y_{l}^{J b}\right) \Omega_{c l j i}\right. \\
& \left.\quad+\left(X_{j}^{I b} Y_{l}^{J c}+X_{j}^{I c} Y_{l}^{J b}\right) \Omega_{c i j l}\right]+\sum_{j l c d}\left[\left(X_{l}^{I d} X_{j}^{J c}+Y_{j}^{I c} Y_{l}^{J d}\right) \Xi_{c l j d b i}\right. \\
& \left.\quad+\left(X_{l}^{I d} Y_{j}^{J c}+X_{j}^{I c} Y_{l}^{J d}\right) \Xi_{c d j l b i}\right]
\end{aligned}
$$




$$
\begin{aligned}
& L_{b i}^{(2)}=\sum_{p q} \Omega_{p b q i} D_{q p}^{I J}+\sum_{j c d}\left[\left(X_{j}^{I c} X_{i}^{J d}+Y_{i}^{I d} Y_{j}^{J c}\right) \Omega_{c b j d}\right. \\
& \left.\quad+\left(Y_{j}^{I c} X_{i}^{J d}+Y_{i}^{I d} X_{j}^{J c}\right) \Omega_{c c j j b}\right]-\sum_{j l c}\left[\left(X_{j}^{I c} X_{l}^{J b}+Y_{l}^{I b} Y_{j}^{J c}\right) \Omega_{c l j i}\right. \\
& \left.\quad+\left(Y_{j}^{I c} X_{l}^{J b}+Y_{l}^{I b} X_{j}^{J c}\right) \Omega_{c i j j}\right]+\sum_{j l c d}\left[\left(X_{j}^{I c} X_{l}^{J d}+Y_{l}^{I d} Y_{j}^{J c}\right) \Xi_{c l j d b i}\right. \\
& \left.\quad+\left(Y_{j}^{I c} X_{l}^{J d}+Y_{l}^{I d} X_{j}^{I c}\right) \Xi_{c d j b i l}\right]
\end{aligned}
$$

and $L_{b i}^{(1)}+L_{b i}^{(2)}=L_{b i}$. In the end, we conclude that, just as for the case of TDHF, ${ }^{41}$ our TD-DFT/RPA derivative couplings agree with the $\mathrm{CM}$ equality near an excited crossing $(\Delta E \rightarrow 0)$ in the limit of an infinite atomic orbital basis.

\section{CONCLUSION}

We have developed an analytic gradient theory for the derivative couplings between TD-DFT/RPA excited states based on pseudo-wavefunctions. Our solution matches the first (timeindependent) formalism of $\mathrm{Li}$ and $\mathrm{Liu}^{38}$ but disagrees with the formalism of Tavernelli et al. ${ }^{34}$ Our final expressions have been implemented numerically and validated against finite-difference for the $S_{1} / S_{5}$ derivative coupling of formaldehyde. For all three types of $x c$ functionals (B3LYP, $\omega$ B97, and $\omega$ B97X) checked, the difference between our result and the finite-difference data is less than $10^{-4} a_{0}^{-1}$.

To help justify our pseudo-wavefunction theory for both TD-HF and TD-DFT/RPA, we first calculated the derivative couplings around the $S_{1} / S_{2}$ CI of protonated formaldimine. The calculations showed that (i) the full-DC vectors recover the correct branching plane as well as the right orientation for both methods and (ii) the full-DC vectors perfectly reproduce the expected Berry phase behavior for a loop around the CI. We have also emphasized that orbital response is essential to recover these two properties. Second, we have shown that our final expression is consistent with the Chernyak-Mukamel equality and time-dependent response theory near an excited crossing. Looking forward, it will be interesting to see how the derivative couplings presented here match with analytical work based exclusively on response theory, i.e., the second formalism presented by Li and Liu. ${ }^{38}$ In the meanwhile, our derivative couplings should find immediate use in nonadiabatic calculations and studies of electronic relaxation.

\section{APPENDIX A: HELLMANN-FEYNMAN DERIVATIVE COUPLINGS FOR TD-DFT/RPA}

As illustrated in ref 41., derivative couplings for TD-HF can also be expressed in the form of a Hellmann-Feynman expression:

$$
\begin{aligned}
\mathbf{d}_{I J}^{\mathrm{TDHF}[Q]}= & \frac{1}{E_{J}-E_{I}} \sum_{i j a b}\left\{\left(X_{i}^{I a} X_{j}^{J b}+Y_{i}^{I a} Y_{j}^{J b}\right)\left\langle\Phi_{i}^{a}\left|\tilde{A}^{[Q]}\right| \Phi_{j}^{b}\right\rangle\right. \\
& +\left[\left(X_{i}^{I a} Y_{j}^{J b}+Y_{i}^{I a} X_{j}^{J b}\right)\left\langle\Phi_{i j}^{a b} \tilde{B}^{[Q]} \mid \Phi_{\mathrm{HF}}\right\rangle\right\}
\end{aligned}
$$

Here $\tilde{A}$ and $\tilde{B}$ are defined as

$$
\begin{aligned}
& \tilde{A}=\mathcal{P}_{1} \hat{H} \mathcal{P}_{1} \\
& \tilde{B}=\mathcal{P}_{2} \hat{H} \mathcal{P}_{0}
\end{aligned}
$$

where $\hat{H}$ is the TD-HF Hamiltonian and $\mathcal{P}_{n}$ are configuration interaction projection operators:

$$
\begin{aligned}
& \mathcal{P}_{0}=\left|\Phi_{\mathrm{HF}}\right\rangle\left\langle\Phi_{\mathrm{HF}}\right| \\
& \mathcal{P}_{1}=\sum_{i a}\left|\Phi_{i}^{a}\right\rangle\left\langle\Phi_{i}^{a}\right| \\
& \mathcal{P}_{2}=\frac{1}{4} \sum_{i j a b}\left|\Phi_{i j}^{a b}\right\rangle\left\langle\Phi_{i j}^{a b}\right|
\end{aligned}
$$

Note that TD-DFT/RPA is a natural extension of TD-HF and therefore one can construct Hellmann-Feynman derivative couplings for TD-DFT/RPA by simply replacing $\hat{H}$ in eqs $\mathrm{A} 2$ and $\mathrm{A} 3$ with the $\mathrm{Kohn}-\mathrm{Sham}$ response operator $O_{\mathrm{KS}}$ (eq 17) and using the Kohn-Sham orbitals to construct the projection operators.

\section{AUTHOR INFORMATION}

\section{Corresponding Author}

*E-mail: subotnik@sas.upenn.edu.

\section{Notes}

The authors declare no competing financial interest.

\section{ACKNOWLEDGMENTS}

J.E.S. thanks Filipp Furche for very stimulating conversations and his suggestion to compare our results versus the Chernyak-Mukamel equality. This work was supported by NSF CAREER Grant CHE-1150851; J.E.S. also acknowledges an Alfred P. Sloan Research Fellowship and a David and Lucile Packard Fellowship.

\section{REFERENCES}

(1) Ball, M. A.; McLachlan, A. D. Time-Dependent Hartree-Fock Theory. Mol. Phys. 1963-1964, 7, 501-513.

(2) Altick, P. L.; Glassgold, A. E. Correlation Effects in Atomic Structure Using the Random-Phase Approximation. Phys. Rev. 1964, 133, A632-A646.

(3) Dunning, T. H.; McKoy, V. Nonempirical Calculations on Excited States: The Ethylene Molecule. J. Chem. Phys. 1967, 47, 17351747.

(4) Lukman, B.; Ažman, A. Application of the RPA Method in the Semi-Empirically Treated Molecular System. Mol. Phys. 1969, 16, 201-202.

(5) Ostlund, N.; Karplus, M. The Self-Consistent Random Phase Approximation. Chem. Phys. Lett. 1971, 11, 450-453.

(6) Miura, M.; Aoki, Y.; Champagne, B. Assessment of TimeDependent Density Functional Schemes for Computing the Oscillator Strengths of Benzene, Phenol, Aniline, and Fluorobenzene. J. Chem. Phys. 2007, 127, 084103.

(7) Heßelmann, A.; Görling, A. Random-Phase Approximation Correlation Methods for Molecules and Solids. Mol. Phys. 2011, 109, 2473-2500.

(8) Dreuw, A.; Head-Gordon, M. Single Reference Ab Initio Methods for the Calculation of Excited States of Large Molecules. Chem. Rev. 2005, 105, 4909.

(9) Casida, M. In Recent Advances in Density Funtional Methods; Chong, D. P., Ed.; World Scientific: 1995; Vol. 1, pp 155-192.

(10) Liu, J.; Liang, W. Analytical Approach for the Excited-State Hessian in Time-Dependent Density Functional Theory: Formalism, Implementation, and Performance. J. Chem. Phys. 2011, 135, 184111.

(11) Terasaka, T.; Matsushita, T. Spin-Projected Random-PhaseApproximation. Chem. Phys. Lett. 1969, 4, 384-388.

(12) Bouman, T. D.; Knobeloch, M. A.; Bohan, S. An Ab Initio Random Phase Approximation Study of the Excited-State Intramolecular Proton Transfer in 3-Hydroxychromone. J. Phys. Chem. 1985, 89, 4460-4464. 
(13) Gatti, C.; Polezzo, S.; Fantucci, P. A Random Phase Approximation Study of the Absorption Spectrum of $\mathrm{Na}_{8}$. Chem. Phys. Lett. 1990, 175, 645-654.

(14) Petke, J. D.; Maggiora, G. M.; Christoffersen, R. E. Ab Initio Configuration Interaction and Random Phase Approximation Calculations of the Excited Singlet and Triplet States of Uracil and Cytosine. J. Phys. Chem. 1992, 96, 6992-7001.

(15) Vracko, M. G.; Zaider, M. A Study of Excited States in TransPolyacetylene in the Hartree-Fock, Tamm-Dancoff, and RandomPhase Approximation. Int. J. Quantum Chem. 1993, 47, 119-127.

(16) Ortiz, J. V. One-Electron Density Matrices and Energy Gradients in the Random Phase Approximation. J. Chem. Phys. 1994, 101, 6743-6749.

(17) Hirsch, J. G.; Civitarese, O.; Reboiro, M. Comparison between Wavefunctions in the Random Phase Approximation, Renormalized Random Phase Approximation, and Self-Consistent Random Phase Approximation Methods. Phys. Rev. C 1999, 60, 024309.

(18) Furche, F. Molecular Tests of the Random Phase Approximation to the Exchange-Correlation Energy Functional. Phys. Rev. B 2001, 64, 195120.

(19) Chernyak, V.; Mukamel, S. Density-Matrix Representation of Nonadiabatic Couplings in Time-Dependent Density Functional (TDDFT) Theories. J. Chem. Phys. 2000, 112, 3572.

(20) Baer, R. Non-Adiabatic Couplings by Time-Dependent Density Functional Theory. Chem. Phys. Lett. 2002, 364, 75-79.

(21) Hu, C.; Hirai, H.; Sugino, O. Nonadiabatic Couplings from Time-Dependent Density Functional Theory: Formulation in the Casida Formalism and Practical Scheme within Modified Linear Response. J. Chem. Phys. 2007, 127, 064103.

(22) Hu, C.; Hirai, H.; Sugino, O. Nonadiabatic Couplings from Time-Dependent Density Functional Theory. II. Successes and Challenges of the Pseudopotential Approximation. J. Chem. Phys. 2008, 128, 154111.

(23) Hu, C.; Sugino, O.; Hirai, H.; Tateyama, Y. Nonadiabatic Couplings from the Kohn-Sham Derivative Matrix: Formulation by Time-dependent Density-Functional Theory and Evaluation in the Pseudopotential Framework. Phys. Rev. A 2010, 82, 062508.

(24) Hu, C.; Sugino, O.; Tateyama, Y. All-Electron Calculation of Nonadiabatic Couplings from Time-Dependent Density Functional Theory: Probing with the Hartree-Fock Exact Exchange. J. Chem. Phys. 2009, 131, 114101.

(25) Send, R; Furche, F. First-Order Nonadiabatic Couplings from Time-Dependent Hybrid Density Functional Response Theory: Consistent Formalism, Implementation, and Performance. J. Chem. Phys. 2010, 132, 044107.

(26) Yarkony, D. R. On the Consequences of Nonremovable Derivative Couplings 0.1. The Geometric Phase and Quasidiabatic States: A Numerical Study. J. Chem. Phys. 1996, 105, 10456-10461.

(27) Domcke, W.; Yarkony, D. R.; Köppel, H. Conical Intersections: Electron Structure Dynamics and Spectroscopy; World Scientific Publishing Co. Pte. Ltd.: Singapore, 2004.

(28) Matsika, S.; Krause, P. Nonadiabatic Events and Conical Intersections. Annu. Rev. Phys. Chem. 2011, 62, 621-643.

(29) Hu, C.; Sugino, O.; Watanabe, K. Second-Order Nonadiabatic Couplings from Time-Dependent Density Functional Theory: Evaluation in the Immediate Vicinity of Jahn-Teller/Renner-Teller Intersections. J. Chem. Phys. 2011, 135, 074101.

(30) Hu, C.; Komakura, R.; Liand, Z.; Watanabe, K. TDDFT Study on Quantization Behaviors of Nonadiabatic Couplings in Polyatomic Systems. Int. J. Quantum Chem. 2013, 113, 263-271.

(31) Hu, C.; Sugino, O.; Watanabe, K. Performance of TammDancoff Approximation on Nonadiabatic Couplings by Time-Dependent Density Functional Theory. J. Chem. Phys. 2014, 140, 054106.

(32) Levine, B. G.; Ko, C.; Quenneville, J.; Martínez, T. J. Conical Intersections and Double Excitations in Time-Dependent Density Functional Theory. Mol. Phys. 2006, 104, 1039-1051.

(33) Levine, B. G.; Coe, J. D.; Martínez, T. J. Optimizing Conical Intersections without Derivative Coupling Vectors: Application to
Multistate Multireference Second-Order Perturbation Theory (MSCASPT2). J. Phys. Chem. B 2008, 112, 405-413.

(34) Tavernelli, I.; Curchod, B. F. E.; Laktionov, A.; Rothlisberger, U. Nonadiabatic Coupling Vectors for Excited States within TimeDependent Density Functional Theory in the Tamm-Dancoff Approximation and beyond. J. Chem. Phys. 2010, 133, 19.

(35) Tapavicza, E.; Tavernelli, I.; Rothlisberger, U. Trajectory Surface Hopping within Linear Response Time-Dependent Density-Functional Theory. Phys. Rev. Lett. 2007, 98, 023001.

(36) Tavernelli, I.; Tapavicza, E.; Rothlisberger, U. Non-Adiabatic Dynamics Using Time-Dependent Density Functional Theory: Assessing the Coupling Strengths. J. Mol. Struct.: THEOCHEM 2009, 914, 22-29.

(37) Tavernelli, I.; Tapavicza, E.; Rothlisberger, U. Nonadiabatic Coupling Vectors within Linear Response Time-Dependent Density Functional Theory. J. Chem. Phys. 2009, 130, 12.

(38) Li, Z.; Liu, W. First-order Nonadiabatic Coupling Matrix Elements between Excited States: A Lagrangian Formulation at the CIS, RPA, TD-HF, and TD-DFT Level. J. Chem. Phys. 2014, 141, 014110.

(39) Fatehi, S.; Alguire, E.; Shao, Y.; Subotnik, J. E. Analytic Derivative Couplings between Configuration-Interaction-Singles States with Built-in Electron-Translation Factors for Translational Invariance. J. Chem. Phys. 2011, 135, 234105.

(40) Ou, Q.; Fatehi, S.; Alguire, E.; Shao, Y.; Subotnik, J. E. Derivative Couplings between TDDFT Excited States Obtained by Direct Differentiation in the Tamm-Dancoff Approximation. J. Phys. Chem. B, submitted for publication, 2014.

(41) Alguire, E.; Ou, Q.; Subotnik, J. E. Calculating Derivative Couplings between Time-Dependent Hartree-Fock Excited States with Pseudo-Wavefunctions. J. Phys. Chem. B 2014, DOI: 10.1021/ jp505767b.

(42) In physicists' notation, $\Pi_{p q s t}$ is given by eq 15 , where $\langle p q \mid s r\rangle=$ $\iint \mathrm{dr}_{1} \mathrm{~d} \mathbf{r}_{2}\left[\left(\phi_{p}^{*}\left(\mathbf{r}_{1}\right) \phi_{q}^{*}\left(\mathbf{r}_{2}\right) \phi_{s}\left(\mathbf{r}_{1}\right) \phi_{r}\left(\mathbf{r}_{2}\right)\right) /\left|\mathbf{r}_{1}-\mathbf{r}_{2}\right|\right]$.

(43) In this article, for simplicity, we will treat $f_{x c}$ as if it is a strictly local functional $f[\rho(\mathbf{r})]$. Extending to generalized gradient approximation (GGA) functionals $f[\rho(\mathbf{r}), \nabla \rho(\mathbf{r})]$ and meta GGAs is straightforward.

(44) Gerratt, J.; Mills, I. M. Force Constants and Dipole Moment Derivatives of Molecules from Perturbed Hartree-Fock Calculations. I. J. Chem. Phys. 1968, 49, 1719-1729.

(45) Pople, J. A.; Krishnan, R.; Schlegel, H. B.; Binkley, J. S. Derivative Studies in Hartree-Fock and M $\phi$ ller-Plesset Theories. Int. J. Quantum Chem. 1979, 16, 225-241.

(46) Handy, N. C.; Schaefer, H. F., III. On the Evaluation of Analytic Energy Derivatives for Correlated Wavefunctions. J. Chem. Phys. 1984, 81, 5031.

(47) Kong, J.; Lee, M. S.; Lee, A. M.; Gwaltney, S. R.; Adams, T. R.; Ochsenfeld, C.; Gilbert, A. T. B.; Kedziora, G. S.; Rassolov, V. A.; Maurice, D. R.; et al. Q-Chem 2.0: A High-performance Ab Initio Electronic Structure Program Package. J. Comput. Chem. 2000, 21, $1532-1548$.

(48) Shao, Y.; Fusti-Molnar, L.; Jung, Y.; Kussmann, J.; Ochsenfeld, C.; Brown, S. T.; Gilbert, A. T. B.; Slipchenko, L. V.; Levchenko, S. V.; O'Neill, D. P.; et al. Advances in Methods and Algorithms in a Modern Quantum Chemistry Program Package. Phys. Chem. Chem. Phys. 2006, 8, 3172-3191.

(49) Lee, C.; Yang, W.; Parr, R. G. Development of the Colle-Salvetti Correlation-Energy Formula into a Functional of the Electron Density. Phys. Rev. B 1988, 37, 758-789.

(50) Becke, A. D. A New Mixing of Hartree-Fock and Local Density Functional Theories. J. Chem. Phys. 1993, 98, 1372-1377.

(51) Chai, J. D.; Head-Gordon, M. Systematic Optimization of LongRange Corrected Hybrid Density Functionals. J. Chem. Phys. 2008, $128,084106$.

(52) Ou, Q.; Subotnik, J. E. Electronic Relaxation in Benzaldehyde Evaluated via TD-DFT and Localized Diabatization: Intersystem Crossings, Conical Intersections, and Phosphorescence. J. Phys. Chem. C 2013, 117, 19839-19849. 
(53) Yarkony, D. R. Energies and Derivative Couplings in the Vicinity of a Conical Intersection Using Degenerate Perturbation Theory and Analytic Gradient Techniques. 1. J. Phys. Chem. A 1997, 101, 4263-4270.

(54) Nelson, T.; Fernandez-Alberti, S.; Chernyak, V.; Roitberg, A. E.; Tretiak, S. Nonadiabatic Excited-State Molecular Dynamics Modeling of Photoinduced Dynamics in Conjugated Molecules. J. Phys. Chem. B 2011, 115, 5402-5414. 